\title{
Intracranial Recordings of Occipital Cortex Responses to Illusory Visual Events
}

\author{
Maartje C. de Jong, ${ }^{1}$ Ralph J. M. Hendriks, ${ }^{1}$ Mariska J. Vansteensel, ${ }^{2}$ Mathijs Raemaekers, ${ }^{2}$ Frans A. J. Verstraten, ${ }^{6,1}$ \\ Nick F. Ramsey, ${ }^{2}{ }^{\circledR}$ Casper J. Erkelens, ${ }^{1}$ Frans S. S. Leijten, ${ }^{2}$ and Raymond van Ee ${ }^{3,4,5}$ \\ ${ }^{1}$ Department of Experimental Psychology, Helmholtz Institute, Utrecht University, 3508 TC Utrecht, The Netherlands, ${ }^{2}$ Department of Neurology and \\ Neurosurgery, Brain Center Rudolf Magnus, University Medical Center Utrecht, 3584 CG Utrecht, The Netherlands, ${ }^{3}$ Philips Research Laboratories, \\ Department of Brain, Body \& Behavior, 5656 AE Eindhoven, The Netherlands, ${ }^{4}$ Laboratory of Experimental Psychology, University of Leuven, 3000 Leuven, \\ Belgium, ${ }^{5}$ Donders Institute, Radboud University, Department of Biophysics, 6500 GL Nijmegen, The Netherlands, and ${ }^{6}$ The University of Sydney, School \\ of Psychology, New South Wales 2006 Sydney, Australia
}

Ambiguous visual stimuli elicit different perceptual interpretations over time, creating the illusion that a constant stimulus is changing. We investigate whether such spontaneous changes in visual perception involve occipital brain regions specialized for processing visual information, despite the absence of concomitant changes in stimulation. Spontaneous perceptual changes observed while viewing a binocular rivalry stimulus or an ambiguous structure-from-motion stimulus were compared with stimulus-induced perceptual changes that occurred in response to an actual stimulus change. Intracranial recordings from human occipital cortex revealed that spontaneous and stimulus-induced perceptual changes were both associated with an early transient increase in high-frequency power that was more spatially confined than a later transient decrease in low-frequency power. We suggest that the observed high-frequency and lowfrequency modulations relate to initiation and maintenance of a percept, respectively. Our results are compatible with the idea that spontaneous changes in perception originate from competitive interactions within visual neural networks.

Key words: ambiguous; binocular rivalry; illusion; intracranial; occipital; visual

\section{Significance Statement}

Ambiguous visual stimuli elicit different perceptual interpretations over time, creating the illusion that a constant stimulus is changing. The literature on the neural correlates of conscious visual perception remains inconclusive regarding the extent to which such spontaneous changes in perception involve sensory brain regions. In an attempt to bridge the gap between existing animal and human studies, we recorded from intracranial electrodes placed on the human occipital lobe. We compared two different kinds of ambiguous stimuli, binocular rivalry and the phenomenon of ambiguous structure-from-motion, enabling generalization of our findings across different stimuli. Our results indicate that spontaneous and stimulus-induced changes in perception (i.e., "illusory" and "real" changes in the stimulus, respectively) may involve sensory regions to a similar extent.

\section{Introduction}

When viewing a stimulus that is constant but ambiguous, an observer perceives changes back and forth between the different

Received Jan. 18, 2015; revised April 19, 2016; accepted May 5, 2016.

Author contributions: M.C.d.J. designed research; M.C.d.J., M.J.V., and F.S.S.L. performed research; F.A.J.V., N.F.R., and R.v.E. contributed unpublished reagents/analytic tools; M.C.d.J., R.J.M.H., and M.R. analyzed data; M.C.d.J., R.J.M.H., M.J.V., M.R., F.A.J.V., C.J.E., F.S.S.L., and R.v.E. wrote the paper.

This work was supported by a Utrecht University High Potential grant (M.C.d.J., R.J.M.H., and R.v.E.), the Netherlands Organization for Scientific Research (M.C.d.J. and F.A.J.V.), the Flemish Methusalem Program (R.v.E., METH/ 08/02 assigned to J. Wagemans), the EU Horizon 2020 Program (R.v.E., HealthPac assigned to J. van Opstal), the Flemish Organization for Scientific Research (R.v.E.), the Technology Foundation STW (M.J.V.), the Technology Program of the Dutch Ministry of Economic Affairs, and Utrecht University (N.F.R., Grant UGT7685). We thank Martin Bleichner, Dora Hermes, Marije ter Wal, and Martijn Barendregt for their assistance and helpful discussion.

The authors declare no competing financial interests. interpretations of the stimulus, while the stimulus is constant. These perceptual changes may be considered illusions, as they originate spontaneously in the mind of the observer, but are experienced as changes of the external stimulus. Here we investigate whether such spontaneous perceptual changes involve regions in the occipital cortex specialized for processing visual information, despite the lack of a concomitant change in the visual stimulus.

Transient activations in the parietal and frontal cortices, rather than the occipital cortex, have been associated with spon-

Correspondence should be addressed to M. C. de Jong, Utrecht University, Experimental Psychology, Heidelberglaan 1, 3508 TC Utrecht, The Netherlands. E-mail: dejongmc@gmail.com.

DOI:10.1523/JNEUROSCI.0242-15.2016

Copyright $\odot 2016$ the authors $\quad 0270-6474 / 16 / 366297-15 \$ 15.00 / 0$ 
A

\begin{tabular}{|c|c|c|c|c|c|c|c|}
\hline participant & age [years] & gender & hemisphere & handedness & used hand & binocular rivalry & 3D-motion rivalry \\
\hline A & 31 & F & R & R & R & yes & yes \\
\hline B & 28 & M & R & L & R & yes & no \\
\hline C & 25 & F & L & L & L & no & yes \\
\hline
\end{tabular}

B
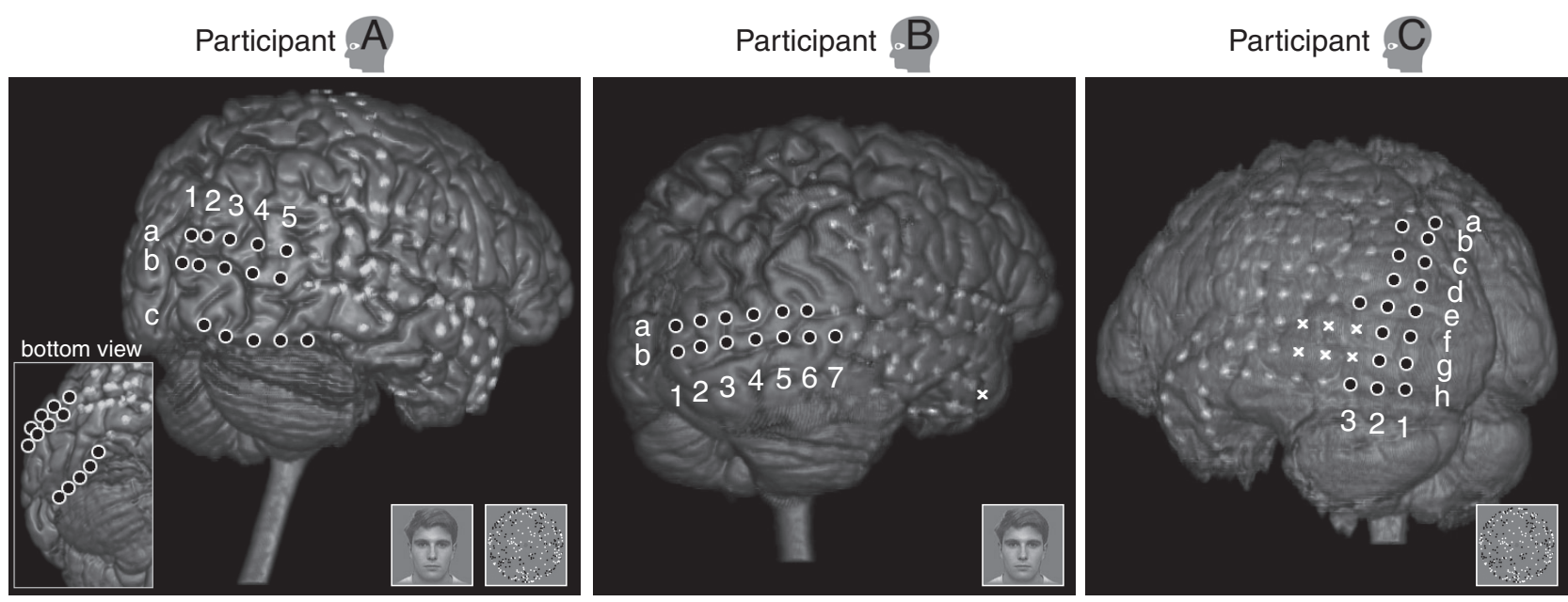

Figure 1. Participants. $A$, Information on the participants. Hemisphere, Sampled hemisphere; handedness, dominant hand; used hand, hand used to operate the button box; L, left; $R$, right; $M$, male; F, female. The small head icons represent the individual participants. $\boldsymbol{B}$, Locations of the electrodes. White circles with black filling indicate occipital electrodes; light gray blobs indicate electrodes on other brain regions (that were not of interest in the present study); white crosses indicate electrodes excluded from the analysis a priori (see Materials and Methods). The white crosses in Participant C covered a temporo-occipital lesion. The white letters $(a-h)$ and numbers $(1-7)$ indicate the names of the occipital electrodes used in the text and in Figure 7 . Miniature stimuli indicate for each participant which experiments were performed.

taneous changes in the perception of a constant ambiguous stimulus (Başar-Eroglu et al., 1996; Kleinschmidt et al., 1998; Lumer et al., 1998; Doesburg et al., 2005; Sterzer and Kleinschmidt, 2007; Ehm et al., 2011; Sandberg et al., 2014). Therefore, it has been argued that spontaneous perceptual changes are initiated in cognitive brain regions and not in occipital regions specialized for visual processing (Leopold and Logothetis, 1999; Sterzer et al., 2009). While viewing an ambiguous stimulus, activity in the occipital cortex has been found to fluctuate with the perceptual state rather than with the changes between percepts, suggesting that the occipital cortex is recruited for maintenance of a percept, but is not involved in the initiation of a new percept (Leopold and Logothetis, 1996; Polonsky et al., 2000; Tong and Engel, 2001; Haynes and Rees, 2005; Brouwer and van Ee, 2007; Maier et al., 2008; de Jong et al., 2012, 2014; Sandberg et al., 2013, 2014). This putative noninvolvement of the occipital cortex in the initiation of new percepts is at odds with evidence indicating that the initiation of spontaneous perceptual changes relies on competitive interactions in the occipital cortex between visual neurons preferentially involved in one of the percepts (mainly psychophysical evidence: for review, see Blake and Logothetis, 2002; but also neuroimaging evidence: Lee et al., 2007; Xu et al., 2016).

Here, we consider two possible reasons why many previous studies have not detected transient modulations of occipital activity in association with spontaneous changes in perception. First, up until now investigations of occipital activity during ambiguous visual stimulation have focused on sustained modulations reflecting the perceptual state (Leopold and Logothetis, 1996; Tong and Engel, 2001), potentially overlooking transient modulations occurring at the moment of a perceptual change. Second, the extracranial recording techniques used on humans in previous studies may not be well suited for detecting spatially localized transients in neural activity (Privman et al., 2007; Jerbi et al., 2009; Crone et al., 2011; Lachaux et al., 2012). We used recordings from intracranial electrodes implanted in epilepsy patients to investigate the extent to which occipital activity is transiently modulated in association with spontaneous and stimulus-induced perceptual changes (i.e., illusory and real changes in the stimulus, respectively).

Our intracranial recordings indeed showed transient modulations of occipital activity associated with spontaneous perceptual changes that occurred without a concomitant change in the visual input. Moreover, these modulations were similar to those associated with stimulus-induced changes in perception. We discuss whether our findings are compatible with a role of the occipital cortex in the spontaneous initiation of a new percept in the absence of a change in stimulation.

\section{Materials and Methods}

Participants. The study included three patients with intractable epilepsy (two female, one male) who underwent chronic subdural electrocorticography that included coverage of the occipital lobe for clinical reasons (Fig. 1). All participants had normal or corrected-to-normal vision and gave written informed consent before participation. The experiments were approved by the Medical Ethical Committee of the University Medical Center Utrecht and were performed in accordance with the ethical guidelines in the Declaration of Helsinki (World Medical Association, 2000). A fourth participant was excluded from the analysis because of excessive epileptiform activity and recording noise.

Procedure. We presented ambiguous visual stimuli that allowed two mutually exclusive perceptual interpretations, thereby enabling separate investigations of the neural underpinnings of visual perception and visual stimulation. To generalize our findings across stimuli, we used two 


\section{Binocular rivalry}

A

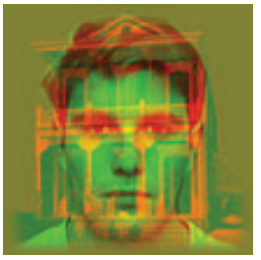

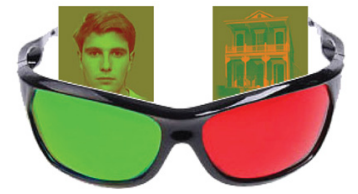

B

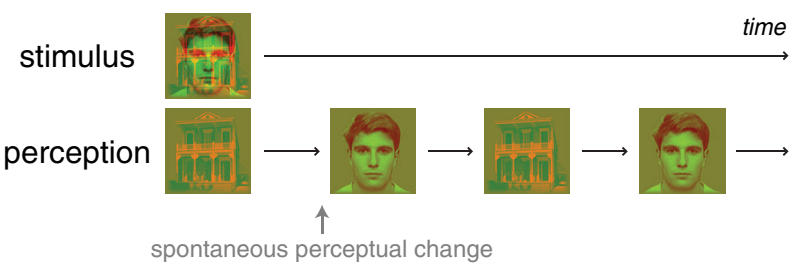

Unambiguous face/house stimulus

C
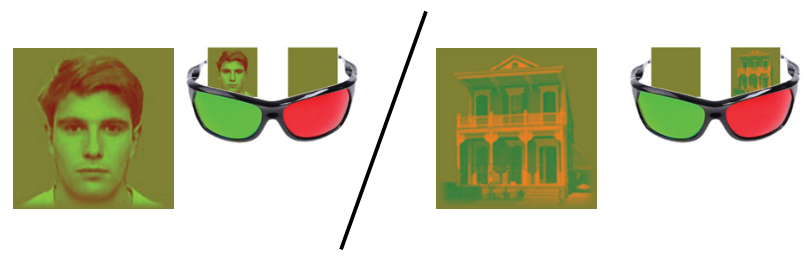

D

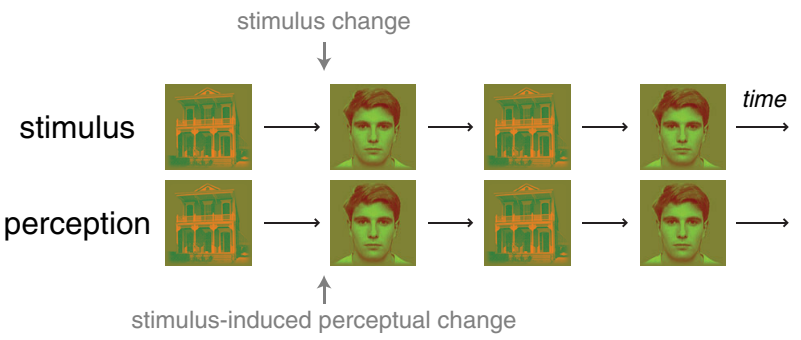

\section{D-motion rivalry}

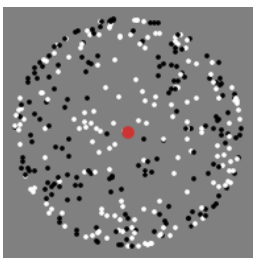

depth cue in

motion of dots

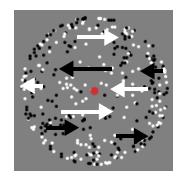

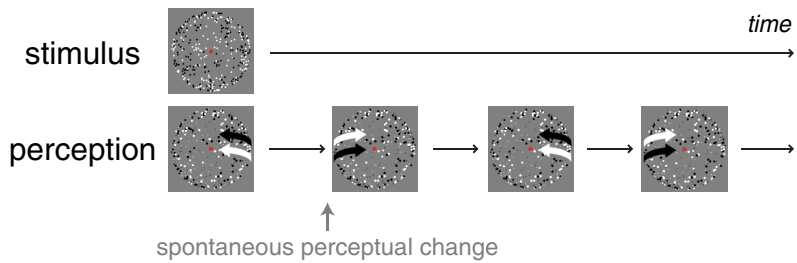
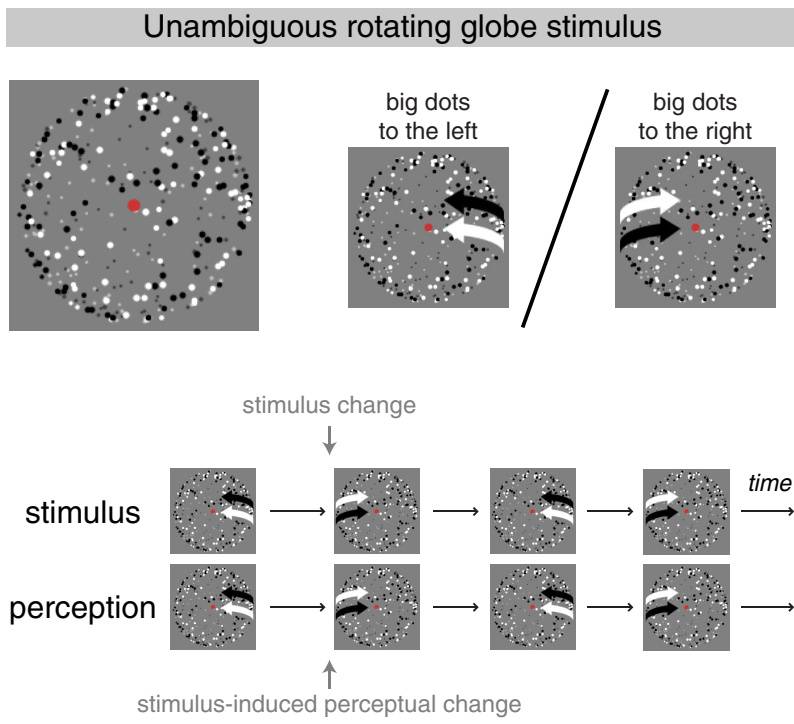

Figure 2. Stimuli. A, Ambiguous stimuli. In the binocular rivalry experiment (left column), one of the eyes was presented with a face image and the other eye with a house image by means of anaglyph glasses. In the 3D-motion rivalry experiment (right column) the 3D interpretation of a virtual globe was ambiguous, as it could be perceived rotating leftward or rightward. B, Spontaneous perceptual changes (i.e., illusory stimulus changes) occurred between two equally likely interpretations of the ambiguous, but constant, stimulus. C, Unambiguous stimuli. To elicitstimulus-induced perceptual changes we presented two alternating unambiguous stimuli that each resembled one of the possible interpretations of the ambiguous stimuli. In the binocular rivalry experiment (left column), these were a face image and a house image. In the 3D-motion rivalry experiment, we reversed the motion direction of the dots and added depth cues (size and contrast modulation). $\boldsymbol{D}$, Stimulus-induced perceptual changes occurred in response to the physical ("real") changes in the unambiguous stimulus. The spontaneous (B) and stimulus-induced perceptual changes were similar in appearance, but only the latter was associated with an actual change in the visual input.

different types of ambiguous stimuli. The first was a binocular rivalry stimulus, including two different (incompatible) images presented to the two eyes (Blake and Logothetis, 2002; Tong et al., 2006), in this case a face and a house image (Fig. $2 A$, left column). The second stimulus was a virtual rotating globe with ambiguous rotation direction. Here both eyes saw the same image, but there was a conflict in the threedimensional (3D) interpretation of the motion in the stimulus (Wallach and O'Connell, 1953; Braunstein, 1977). We will refer to this as 3Dmotion rivalry (Fig. $2 \mathrm{~A}$, right column). When viewing an ambiguous stimulus, an observer's perception spontaneously alternates even though the stimulus itself remains unchanged (Fig. 2B).

To check whether our findings are specific for ambiguous stimulation, we compared both ambiguous stimuli with unambiguous stimuli that were similar in appearance (Fig. 2C). Alternating presentation of the two unambiguous stimuli, each resembling one of the interpretations of the ambiguous stimulus, elicited changes in perception that were designed to be nearly indistinguishable in appearance to the spontaneous changes perceived in the ambiguous stimulus. The important difference is that here the stimulus actually changed, i.e., the perceived changes in the unambiguous stimulus were associated with a change in the physical stimulus (Fig. 2D), whereas the changes in the ambiguous stimulus originated in the mind of the observer (Fig. $2 B)$. The participants were not informed about this difference in stimulation.

During the experiments, participants sat in a semirecumbent position in a hospital bed in a private room. A computer screen $(60 \mathrm{~Hz}$ refresh rate) was positioned in front of the participant at a viewing distance of $\sim 85 \mathrm{~cm}$. The stimuli were presented in the center of the screen using Presentation (Neurobehavioral Systems). The participants reported the identity of each new percept as soon as perception changed by pressing one of two buttons on a button box held in the preferred hand (Fig. 1A). Participant A completed both experiments. Participant B completed only the binocular rivalry experiment because he perceived intermediate percepts during the $3 \mathrm{D}$-motion rivalry experiment (see criteria and ex- 
planation in following paragraphs). Participant C completed only the 3D-motion rivalry experiment because the condition of the participant did not allow for more testing.

Before the start of the experiment the possible perceptual appearances of the stimuli were explained to the participant and care was taken that the participant understood how perception could change irregularly over time. The appearances of the "target" percepts were described, being leftward and rightward rotation for 3D-motion rivalry and the face and house images for binocular rivalry. Regarding binocular rivalry, small stimulus size and low stimulus contrast were used to generate relatively long-lasting percepts and minimize the occurrence of mixture/piecemeal percepts (a mixture of the face and house). We aimed for long-lasting percepts because this makes reporting simpler. The stimuli are described in more detail in the next section. The participants were instructed to report the face or house percept only when this image clearly dominated over the other image, i.e., when little or nothing of the other image was visible. They were also instructed to stop the experiment when the target percepts did not predominate, for example when piecemeal percepts predominated instead. However, both participants that completed the binocular rivalry experiment reported no discernable piecemeal rivalry (in line with pilot tests on healthy volunteers).

Regarding 3D-motion rivalry, the instruction was that the front surface, as a whole, should be perceived closer in depth than the back surface. A slow rotation speed was used to generate relatively long-lasting percepts (Brouwer and van Ee, 2006). It was explained that intermediate percepts could occur, such as blending together of the front and back surface (Hol et al., 2003). The participants were instructed to stop the experiment when they were unable to identify the target percepts or when the target percepts did not predominate. As mentioned above, Participant B did not complete the experiment for this reason. The two remaining participants reported no difficulty with identifying the target percepts.

An experimental session consisted of four 2 min blocks of stimulation interleaved with $10 \mathrm{~s}$ rest, during which only the fixation stimulus was presented on the screen. The blocks of stimulation contained either ambiguous or unambiguous stimulation. Ambiguous and unambiguous blocks were alternately presented. In each experiment, the participants completed two sessions, of which the first started with an unambiguous block and the second with an ambiguous block. The durations of the unambiguous stimuli were based on the percept durations during ambiguous stimulation reported by healthy volunteers in pilot tests.

Stimuli in binocular rivalry experiment. The ambiguous stimulus subtended $2.9^{\circ}$ horizontally and vertically, and consisted of a chromatically filtered image of a face and a house projected at the same location (Fig. $2 A$, left column). By means of red-green anaglyph glasses, one of the eyes was presented with the face image and the other eye with the house image (stereoscopic presentation), without visible "cross talk" between the eyes. The images are courtesy of F. Tong (Tong et al., 2006). The contrast of the original face stimulus was slightly reduced using Photoshop 11.0.2 (Adobe) to achieve similar durations of the face and house percept during ambiguous stimulation (SD of green pixel values in face stimulus was reduced from 42.1 to 36.4 ; adjustment based on pilot testing on healthy volunteers; SD of red pixel values in house stimulus was 44.7). The anaglyph glasses contained custom-made red and green filters, of which the transmission spectrum matched the emission spectrum of the monitor and which resulted in low stimulus contrast on the retina. The low stimulus contrast and small stimulus size ensured relatively long-lasting percepts. The participants wore the anaglyph glasses during the entire experiment.

The images were presented in the center of a binocular pattern of lines extending into the periphery of the visual field to facilitate proper alignment of the eyes (visible by both eyes, covering $15.8^{\circ}$ horizontally and vertically). The binocular lines were presented continuously during the entire session, including the rest periods. The perceptual conflict between the eyes resulted in the alternating dominance of the face and the house image (Levelt, 1966; Blake and Logothetis, 2002). The participants were instructed to fixate on the center of the face/house image and report the perceptual changes they experienced.
To disambiguate the binocular rivalry stimulus, either the face or house image was temporarily removed from the stimulus (Fig. 2C, left column). Observers perceived the remaining image, which, similar to the ambiguous stimulus, was presented only to one eye. Stimulus changes occurred every couple of seconds (42 changes in total during two sessions) and consisted of the alternating presentation of either the face or the house image. The stimuli were created using Photoshop 11.0.2 (Adobe).

Stimuli in 3D-motion rivalry experiment. The ambiguous virtual globe subtended $4.7^{\circ}$ in diameter and consisted of 350 leftward and rightward moving dots presented on a gray background. The dots were black or white, subtended $0.11^{\circ}$ in diameter, had unlimited lifetime, and were perceived as random points on the surface of a virtual globe. The globe was perceived rotating about its vertical axis (every $6.3 \mathrm{~s}$ ), but the direction of rotation was ambiguous: either the front surface was perceived to displace leftward and the back surface rightward or the other way around (Fig. 2A, right column). This ambiguity arose by means of a phenomenon called structure from motion, which refers to a situation in which the $3 \mathrm{D}$ shape of an object is reconstructed from local motion signals, in this case the motion of the dots (Wallach and O'Connell, 1953; Braunstein, 1977). Specifically, the speed of the dots on the screen was fastest in the middle of the stimulus and slowest near the left and right edges (where their motion direction reversed). The sinusoidal speed profile created the $3 \mathrm{D}$ percept. However, there were no depth cues to differentiate the rightward moving surface from the leftward moving surface, so either direction could be perceived to be in front of the other.

Disambiguation of the 3D-motion rivalry stimulus was less straightforward than disambiguation of the binocular rivalry stimulus. We disambiguated the globe by adding two monocular depth cues (Fig. 2C, right column), namely (1) a contrast imbalance: the contrast between the dots and the background was halved for the back surface of the globe compared with the front surface of the globe; and (2) a size imbalance: the size of the dots varied with virtual depth (between 0.05 and $0.15^{\circ}$ in diameter, smaller dots on the back surface). A stimulus change consisted of instantly reversing the motion direction of all dots (44 changes in total during two sessions). The globe stimuli were created using Mathematica (Wolfram Research). During the entire session, a red fixation dot was presented in the center of the screen $\left(0.24^{\circ}\right.$ in diameter $)$ and the participants were instructed to maintain strict fixation on this red dot.

Behavioral analysis. In our analysis we compared the spontaneous changes reported during the ambiguous blocks with the stimulusinduced changes reported during the unambiguous blocks. If reports followed each other within $1000 \mathrm{~ms}$, we excluded the second report, because neural activation related to the earlier report could contaminate the epoch of the second report. If the same report was given twice consecutively, we also excluded the second report. For these reasons, three reports of Participant A (binocular rivalry experiment, ambiguous blocks), two reports of Participant B (ambiguous blocks), and one report of Participant $\mathrm{C}$ (unambiguous block) were excluded. We also excluded the very first report of each block, because this report is associated with the perceived onset of the stimulus rather than a perceived change in the stimulus.

Regarding the unambiguous blocks, we only included correct reports, defined as reports $<3500 \mathrm{~ms}$ after a stimulus change, indicating the stimulus was perceived as intended by the applied disambiguation method. A control analysis in which we used a more conservative cutoff for reaction times ( $<1500 \mathrm{~ms}$ instead of $<3500 \mathrm{~ms}$ ) yielded equivalent results regarding the observed power changes (data not shown), indicating that the used cutoff did not critically influence our results. During the unambiguous blocks of the binocular rivalry experiment, all reports were correct for both participants. During the unambiguous blocks of the 3D-motion rivalry experiment, three reports of Participant A were incorrect $(>3500 \mathrm{~ms}$ ) and 30 reports of Participant $\mathrm{C}$ were incorrect and thus excluded from the analysis. Participant $\mathrm{C}$ experienced spontaneous changes in addition to the stimulus-induced changes during the unambiguous blocks, despite the intended disambiguation of the stimulus.

Electrocorticographic recording and processing. The subchronic electrocorticographic recordings were part of a presurgical assessment to localize the epileptic focus for surgical removal. Electrode grids were 
implanted subdurally ( $2.3 \mathrm{~mm}$ exposed diameter, interelectrode distance $1 \mathrm{~cm}$; Ad-Tech Medical Instrument). We recorded continuously using a 128-channel Micromed system (22 bits; bandpass filter, 0.15-134.4 Hz) at a sampling frequency of $512 \mathrm{~Hz}$. The recorded data were analyzed using in-house-developed Matlab code and the open source Matlab toolbox EEGlab (Delorme and Makeig, 2004). The precise positioning of the electrodes was determined using an anatomical magnetic resonance (MR) imaging scan made before implantation and a computed tomography (CT) scan made after implantation, following the method described by Hermes et al. (2010). Electrodes affected by a temporooccipital lesion in Participant C, as identified by a clinical physicist using the coregistered MR and CT images, were excluded from the analysis (Fig. 1B). The signals of all electrodes were visually inspected for artifacts, leading to one anterior electrode of Participant $B$ being excluded from the analysis because of technical failure (Fig. $1 B$ ). As mentioned above in the Participants section, all data of a fourth participant were excluded after visual inspection by a medical professional because of excessive epileptiform activity and recording noise.

All remaining electrode signals were referenced to their grand average signal. Experimental epochs centered on the moment of the report (button press) were extracted from the continuous data. We used anatomical landmarks to assess which electrodes were located on the surface of the occipital lobe. Specifically, all electrodes posterior of the imaginary line between the preoccipital notch and the parieto-occipital sulcus were classified as occipital electrodes (Fig. 1B). A time-frequency transformation was applied to the occipital electrodes over a frequency range of 2-130 Hz (using a 3-97.5 cycle Morlet wavelet, respectively, tapered with a Hanning window and taking frequency steps of $1 \mathrm{~Hz}$ and time steps of $25 \mathrm{~ms}$ ). There was no true baseline period because we do not know precisely when the spontaneous changes were initiated. Therefore, we normalized the power per epoch, per frequency, relative to the mean power across a broad time window (normalized power equals power/ mean power; normalization window was $5 \mathrm{~s}$ wide and centered on the moment of the report). Perturbations were then averaged across two frequency bands of interest derived from previous studies into stimulusinduced perceptual changes: $3-30$ and $50-130 \mathrm{~Hz}$ (Tallon-Baudry and Bertrand, 1999; Lachaux et al., 2005; Hoogenboom et al., 2006; Wilke et al., 2006; Siegel et al., 2007; Maier et al., 2008; Tallon-Baudry, 2009; Donner and Siegel, 2011).

In none of the participants did the occipital electrodes overlap with the area resected during postimplantation surgery, meaning that the analyzed electrodes were not covering the focus region of the epileptic seizures (the seizure-onset zone). Initially, and unless indicated otherwise, we analyzed the averaged activity of all occipital electrodes (see first part of Results; Figs. 3-5). To see whether the effects observed in the averaged occipital recordings were systematic across electrodes, we then analyzed the recordings of the individual occipital electrodes (see last part of Results; Figs. 6, 7). When referring to individual electrodes, we occasionally make estimations regarding which visual regions were covered by the electrodes. These estimations are speculative since they were not verified by functional mapping experiments, but instead were based on anatomical landmarks and an automated computerized segmentation algorithm (Freesurfer Software Suite). The computerized algorithm provided an estimated location of primary visual cortex (area V1), secondary visual cortex (area V2), and motion-selective mediotemporal region (area V5/ $\mathrm{hMT}+)$.

Peak-locked perturbations and power change area. Considering that the averaged perturbations aligned to the report may be affected by jitter in reaction times (i.e., variation in the time period between the perceptual change and the button press; see Results), we performed two additional analyses largely insensitive to such jitter. We expected activity related to perceptual changes to emerge before the report and then linger on for a while, so we chose for these analyses a time window of interest spanning -1500 to $+500 \mathrm{~ms}$ relative to the report.

First, rather than calculating peak amplitudes, we calculated the areas delimited by the perturbations. Regarding the increase in high-frequency power, we calculated the area below the curve of power over time (and above the horizontal axis that indicated the normalization value, i.e., normalized power equals 1). Regarding the decrease in low-frequency power, we calculated the area above the curve of power over time (and below the horizontal axis). The calculation was done per epoch, across the time window of interest mentioned above. Temporal variation within the time window has no influence on the calculated areas, making this measure largely insensitive to reaction-time jitter. We did not calculate the mean power over the broad time window of interest because we were interested specifically in negative and positive event-related components and, moreover, calculating the mean power would allow the possibility of averaging out components of opposite sign (Luck, 2005). To adjust for noise-related fluctuations, we applied the same calculation to a reference time window spanning 500-2000 ms after the report (Fig. 4A, schematic illustration on bottom right). We subtracted the reference area from the area in the time window of interest (after scaling both areas to the width of the used time windows) and then averaged across epochs. We used a postreport rather than a prereport reference window because there is an unknown delay between the report and the preceding onset of activity related to the perceptual change. Hence, a prereport reference window may be contaminated by early activity related to the build-up of a perceptual change. Statistical analysis was performed using SPSS Statistics 20 (IBM).

For the second analysis aimed at circumventing the influence of reaction-time jitter, we determined, per epoch, per frequency band, the latency of the peak in the power modulation (minimum or maximum amplitude within the time window of interest for negative and positive power fluctuations, respectively) and aligned the epochs in time to these latencies before averaging across epochs. The resulting peak-locked perturbations were used for illustration purposes (Figs. $5 A-D$ ), as they show the perturbations without the influence of reaction-time jitter. The peak latencies themselves were analyzed to obtain an estimate of the relative timing of the low-frequency and high-frequency modulation (Fig. $5 E$, Results). Considering that differences in mean reaction time affect these peak latencies, meaningful comparisons could be made only within participants, within each type of change.

\section{Results}

\section{Behavioral results}

Participants A and B reported spontaneous changes in perception of the binocular rivalry stimulus every 5.9 (4.1) s and every 7.0 (4.6) s on average (medians in parentheses), respectively. Participants $\mathrm{A}$ and $\mathrm{C}$ reported spontaneous changes in perception of the 3D-motion rivalry stimulus every $13.7(14.0)$ and $9.2(8.7) \mathrm{s}$ on average (medians in parentheses), respectively. The unambiguous stimulus durations were set to 10.0 (5.9) and 10.0 (9.8) s on average (medians in parentheses) in the binocular rivalry and 3D-motion rivalry experiment, respectively. The mean reaction times (delay between stimulus change and report) of Participant $\mathrm{A}$ and $\mathrm{B}$ in the binocular rivalry experiment and Participants $\mathrm{A}$ and $\mathrm{C}$ in the 3D-motion rivalry experiment were 830 (829), 741 (627), 1196 (984), and 1308 (1094) ms (medians in parentheses), respectively. The number of analyzed epochs for these participants (in the same order) were $42,42,41$, and 18 regarding stimulus-induced changes, and 78, 60, 32, and 46 regarding spontaneous changes, respectively. Only accurate reports were analyzed (see Materials and Methods).

\section{Analysis of electrocorticographic recordings}

The exact moment that the spontaneous perceptual changes occurred is unknown because they originated in the mind of the participants. Therefore, we aligned the epochs in time to the moment of the manual report (button press). Even though the spontaneous changes were perceptual-and not sensory-in nature, the occipital recordings showed clear event-related responses in both experiments and all participants. Preceding the button press there was a broadband decrease in the power of low frequencies (3-30 Hz; theta, alpha, and beta band) and a broadband increase in the power of high frequencies $(50-130 \mathrm{~Hz}$; higher 

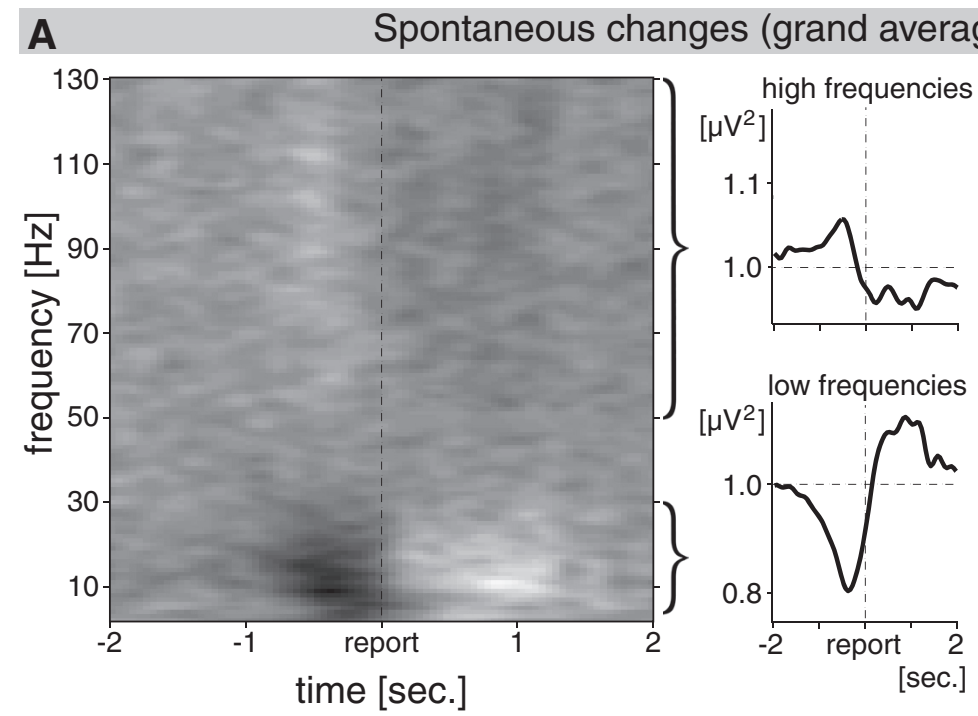

normalized power $\left[\mu \mathrm{V}^{2}\right]$

0.65

$1.0 \quad 1.35$

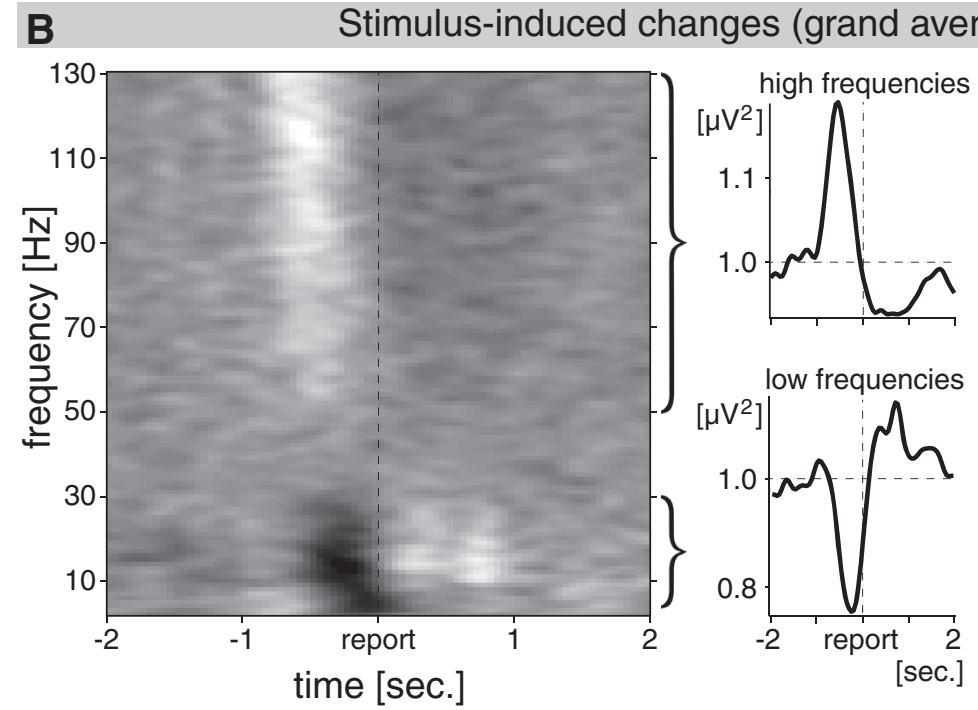

hanges (grand average)
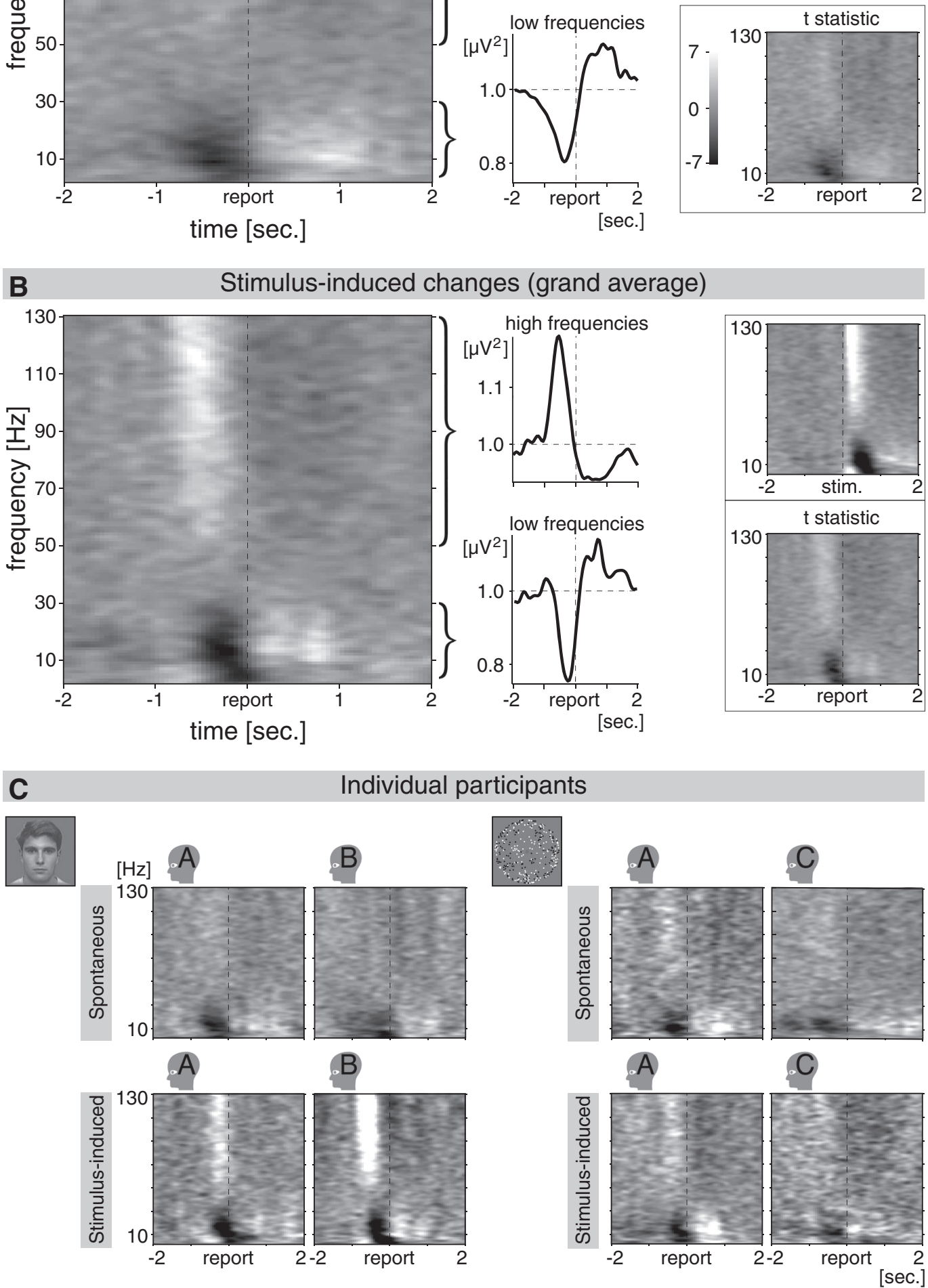

Figure 3. Event-related spectral perturbation recorded from occipital subdural electrodes. $A, 0$ ccipital event-related spectral perturbation (mean of all occipital electrodes) for spontaneous perceptual changes. Data are averaged across participants and experiments ( 4 datasets are averaged, namely 2 participants for each of the 2 experiments). See individual datasets in $\boldsymbol{C}$. The epochs were time-locked to the moment the participant reported the spontaneous change (indicated as "report" on the time axis). Gray scales indicate the normalized power (Figure legend continues.) 
gamma band; Fig. 3A). A similar event-related spectral perturbation was observed for stimulus-induced changes in perception (Fig. $3 B$ ). This pattern of results was present in each individual participant (Fig. $3 C$ ) and was also observed in the averaged $t$ statistics reflecting the variance across epochs (Fig. $3 A, B$, bottom right insets).

Note that the averaged perturbation reflecting stimulusinduced changes appeared more spread out in time when time-locked to the report (Fig. $3 B$, graph on the left) than when time-locked to the stimulus change (Fig. $3 B$, inset in upper right corner). This could reflect jitter in reaction times (interval between stimulus change and report of perception of the change), affecting the report-locked and not the stimulus-locked perturbation. Since there is no stimulus change associated with the spontaneous perceptual changes, statistical analyses require a measure that is largely insensitive to reaction-time jitter. Therefore, we analyzed the area of a power change (area under the curve for power increase; area above the curve for power decrease), rather than the peak amplitude of a power change. The area was calculated per epoch, across a (report-locked) time window of interest (see Materials and Methods; Fig. 4A, schematic illustration on bottom right). For this analysis, as well as for all other analyses described below, we averaged the spectral perturbations within the $3-30$ and $50-130 \mathrm{~Hz}$ bands (Fig. $3 A, B$, line graphs) and we used the recordings of individual participants, without averaging across participants (Figs. 4-7 thus present each participant individually).

For illustration purposes only, we also obtained a visual representation of the perturbations unaffected by reaction-time jitter by means of aligning epochs to peak latency before averaging across epochs (Fig. 5A-D; peak latency refers to latency of minimum and maximum amplitude for the low-frequency and highfrequency modulation, respectively).

\section{Low-frequency power decrease}

In all participants and both experiments, a highly significant lowfrequency power decrease was observed for both types of changes (Fig. 4A, $C$; type-of-change $\times$ participant ANOVA performed per experiment on the area of the power decrease; intercept: both $F>11,117.7$, both $p \approx 0$ ). The perturbations appeared more spread out for spontaneous than for stimulus-induced changes, particularly in the binocular rivalry experiment. However, the

\section{$\leftarrow$}

(Figure legend continued.) (see scale in upper right corner). Despite the lack of a concomitant change in the visual input, spontaneous perceptual changes were associated with transient modulations in the occipital cortex, a part of the brain specialized for processing visual information. Preceding the report, there was an increase in the power of high frequencies (higher gamma band; see upper curly bracket and line graph) and a slightly later decrease in the power of low frequencies (theta, alpha, and beta band; see bottom curly bracket and line graph). Further analysis was performed on the average perturbation across these two broad frequency bands (as in the line graphs on the right). The inset in the bottom right corner shows the averaged $t$ statistics for the same data, calculated for the individual datasets (mean occipital perturbation across epochs) and then averaged across the four datasets. Sec., Seconds. B, OCcipital event-related spectral perturbation for stimulus-induced perceptual changes, resembling the perturbation for spontaneous changes presented in $\boldsymbol{A}$ (average of 4 datasets, as in $\boldsymbol{A}$ ). Averaged $t$ statistics are presented in the bottom right corner. Layout and details as in $\boldsymbol{A}$. The inset in the upper right corner presents the same spectral perturbation as in the main graph, but time-locked to the change in the unambiguous stimulus (stim.) instead of the report. Contrary to the report-locked perturbation, the stimulus-locked perturbation is not affected by jitter in reaction times. C, Occipital event-related spectral perturbation for spontaneous (upper row) and stimulus-induced (bottom row) perceptual changes for each individual participant, averaged across epochs (on average 45 epochs per graph; see Results). Binocular and 3D-motion rivalry experiments are presented on the left and right, respectively. differences observed in the report-locked perturbations (Fig. $4 A, C$ ) were likely due to jitter in reaction times, as they were not present in the peak-locked perturbations (Fig. $5 \mathrm{~A}, \mathrm{C}$ ). Correspondingly, the areas of the report-locked modulations were similar in magnitude for both types of changes in all participants and both experiments (Fig. 4A, $C$, bar charts; type of change: both $F \leq$ 0.1 , both $p>0.7$; participant: both $F \leq 1.6$, both $p>0.2$; type of change $\times$ participant: both $F \leq 1.6$, both $p>0.2$ ). Spontaneous and stimulus-induced changes were thus associated with a similar transient drop in low-frequency power in the occipital cortex, even though the stimulus was changed in the latter but not in the former.

When low-frequency sub-bands were tested separately, we also found no differences between spontaneous and stimulusinduced changes (bands tested: theta, 3-8 Hz; alpha, 9-13 Hz; lower beta, 14-20 Hz; higher beta, 21-30 Hz; data not shown).

\section{High-frequency power increase}

In the $3 \mathrm{D}$-motion rivalry experiment, the increase in high-frequency power was similar in magnitude for spontaneous and stimulusinduced changes (Fig. 4D; type-of-change $\times$ participant ANOVA performed per experiment on the area of the power increase; type of change: $F_{(1,133)}=0.0, p=1$; participant: $F_{(1,133)}=2.3, p=0.1$; type of change $\times$ participant: $F_{(1,133)}=0.4, p=0.5$; intercept: $F_{(1,133)}=$ 38,743.0, $p \approx 0$; Fig. $5 D$, peak-locked perturbations).

In the binocular rivalry experiment, by contrast, the highfrequency modulation appeared larger for the stimulus-induced changes than for the spontaneous changes in the report-locked (Fig. $4 B$ ) as well as the peak-locked (Fig. 5B) perturbations, suggesting this difference was unrelated to reaction-time jitter. Correspondingly, the area of the report-locked perturbations was larger for spontaneous than for stimulus-induced changes (effect of type of change: $F_{(1,218)}=40.0, p \approx 0$; participant: $F_{(1,218)}=0.4$, $p=0.5)$. In Participant A, the high-frequency power increase for spontaneous changes in perception during binocular rivalry was small but significant, whereas in Participant B it was not significant (type of change $\times$ participant: $F_{(1,218)}=11.1, p=0.001$; Participant A, type of change: $F_{(1,118)}=5.1, p=0.026$; Participant $\mathrm{B}$, type of change: $F_{(1,100)}=41.2, p \approx 0$; Participant $\mathrm{A}$, spontaneous changes: $t_{(77)}=2.5, p=0.015$; Participant $\mathrm{B}$, spontaneous changes: $\left.t_{(59)}=-0.9, p=0.4\right)$.

\section{Peak latency of low-frequency and high-frequency power change}

We compared the peak latencies of the low-frequency and highfrequency modulation (frequency band $x$ type of change ANOVA performed per participant; we were not interested in the main effect of type of change, as it reflects differences in reaction times; see Materials and Methods) and found that the increase in high-frequency power peaked earlier than the decrease in lowfrequency power in the binocular rivalry experiment (Fig. 5E; frequency band: both $F>5.6$, both $p<0.02$ ). In the 3D-motion rivalry experiment the same trend was present (Fig. $5 E$ ). In both experiments there was no significant difference between spontaneous and stimulus-induced changes regarding this latency difference (frequency band $\times$ type of change: all $F \leq 2.6$, all $p>0.1$ ).

Since latency estimates could be influenced by the width of the wavelet used for the time-frequency transformation (which was twice as broad for the lowest than for the highest frequency; see Materials and Methods), we recalculated the latencies of the peaks using the same wavelet width for all frequencies $(1500 \mathrm{~ms}$ for all). This analysis yielded equivalent results regarding the averaged occipital recording (data not shown). We also analyzed 


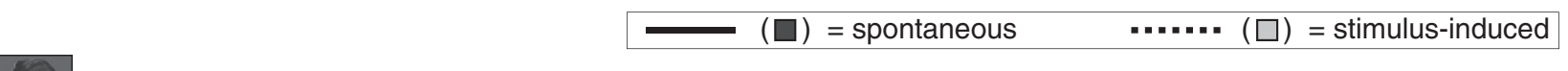

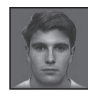

Low frequency band $(3-30 \mathrm{~Hz})$

A
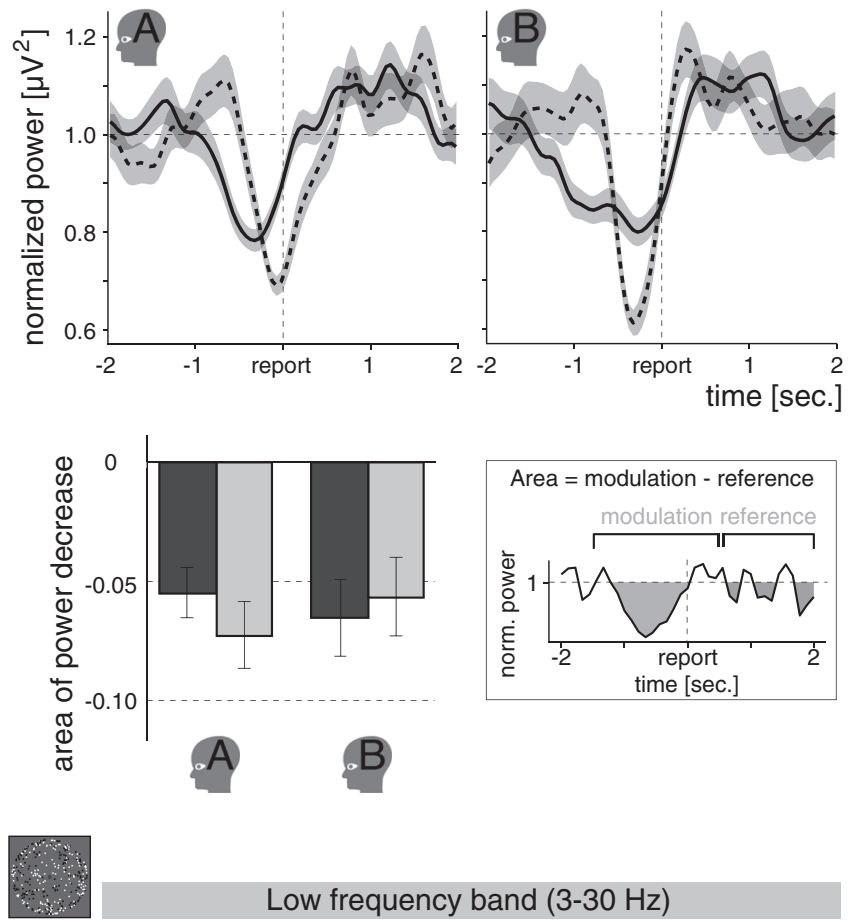

Low frequency band $(3-30 \mathrm{~Hz})$

C
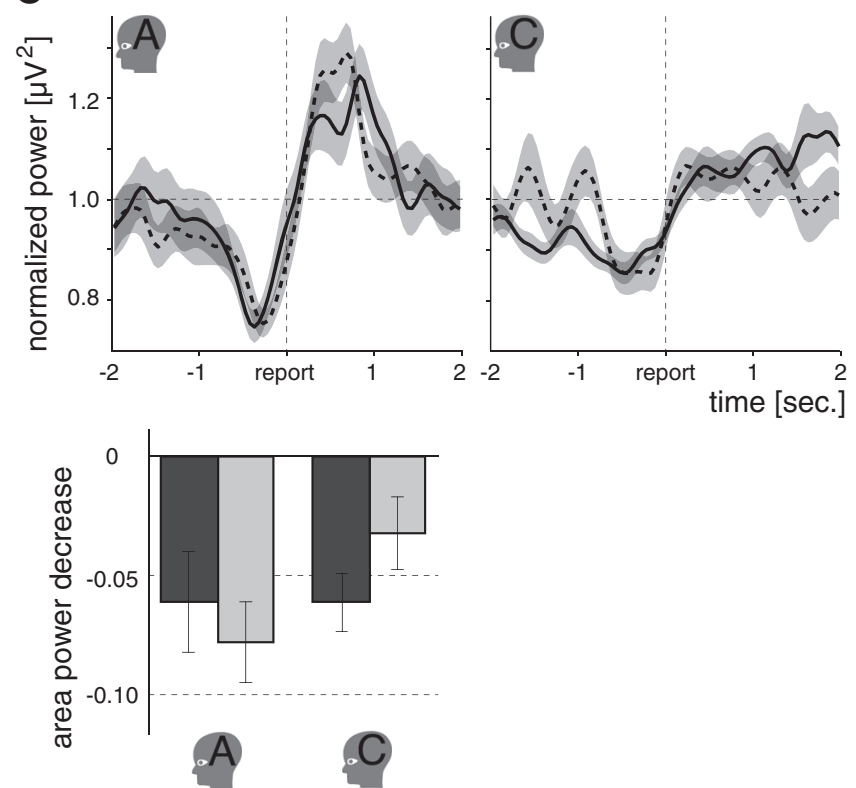

High frequency band $(50-130 \mathrm{~Hz})$

B
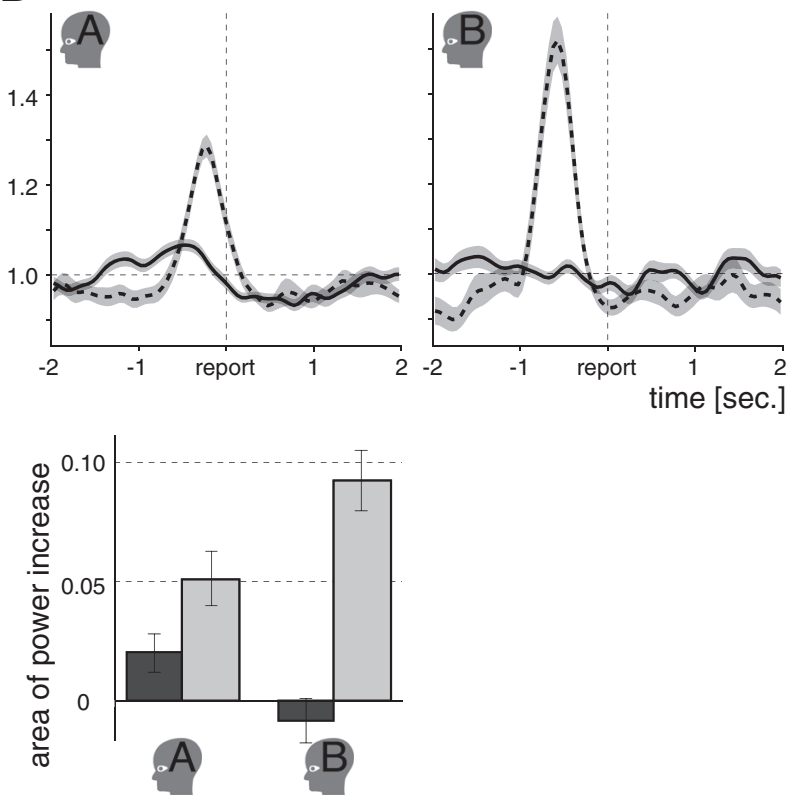

D
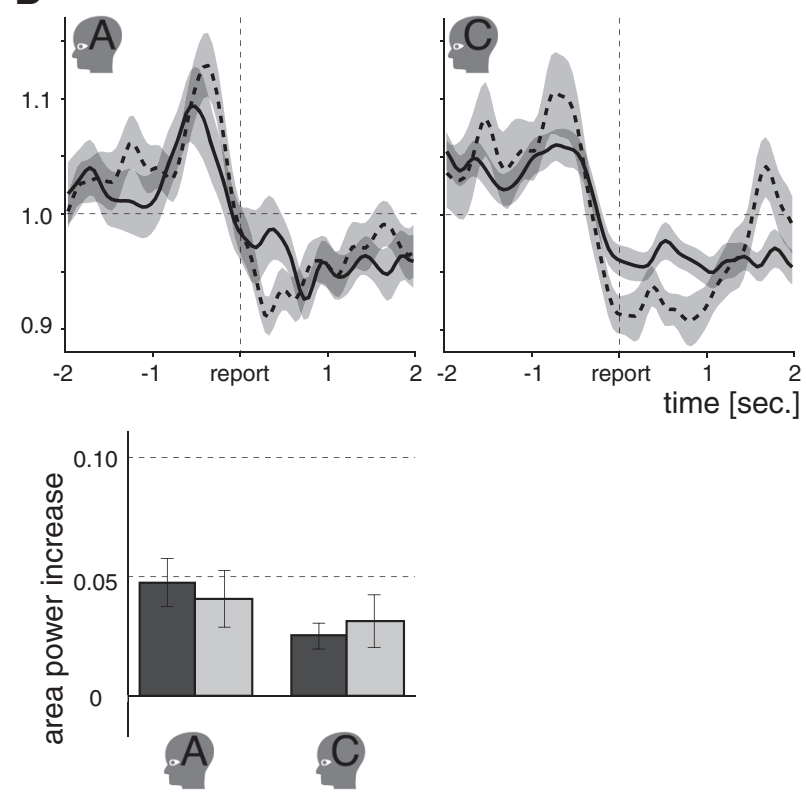

Figure 4. Spectral perturbations for individual participants. $A$, Top, Spectral perturbations for the low-frequency band of interest $(3-30 \mathrm{~Hz})$ in the binocular rivalry experiment, averaged across epochs for Participants A and B separately (presented on the left and right, respectively). Each line on average represents 56 epochs. The perturbations are time-locked to the moment a spontaneous (solid lines) or stimulus-induced (dashed lines) change was reported (indicated as "report" on the time axis). Spontaneous as well as stimulus-induced perceptual changes were associated with an occipital low-frequency power decrease, even though the stimulus was changed in the latter, but not in the former. Shading indicates \pm SEM across epochs. Bottom, Area of the low-frequency power decrease for the individual participants of the binocular rivalry experiment ( \pm SEM across epochs; see schematic illustration on the right). Whereas the perturbations may be influenced by jitter in reaction times, the areas are insensitive to such jitter. Spontaneous (dark gray bars) and stimulus-induced (light gray bars) changes were associated with a similar drop in low-frequency power. Miniatures of the stimuli indicate the respective experiments. Small head icons indicate participants. $\boldsymbol{B}$, Top, Spectral perturbations for the high-frequency band of interest (50-130 $\mathrm{Hz})$ in the binocular rivalry experiment, averaged across epochs (on average 56 epochs per line) for Participants $A$ and B separately (presented on the left and right, respectively). Preceding the report there was an increase in high-frequency power for stimulus-induced changes that was less clear for spontaneous changes. Bottom, Areas of the high-frequency power increases for the individual participants of the binocular rivalry experiment. The high-frequency power increase was smaller for the spontaneous than for the stimulus-induced changes. Details as in $\boldsymbol{A}$. C, Top, Spectral perturbations for the low-frequency band of interest in the 3D-motion rivalry experiment, averaged across epochs (on average 34 epochs per line) for Participants A and ( separately (presented on the left and right, respectively). As in the binocular rivalry experiment $(\boldsymbol{A})$, spontaneous as well as stimulus-induced changes elicited a decrease in low-frequency power. Bottom, (Figure legend continues.) 


\section{Low-frequency perturbations (time-locked to peak)}
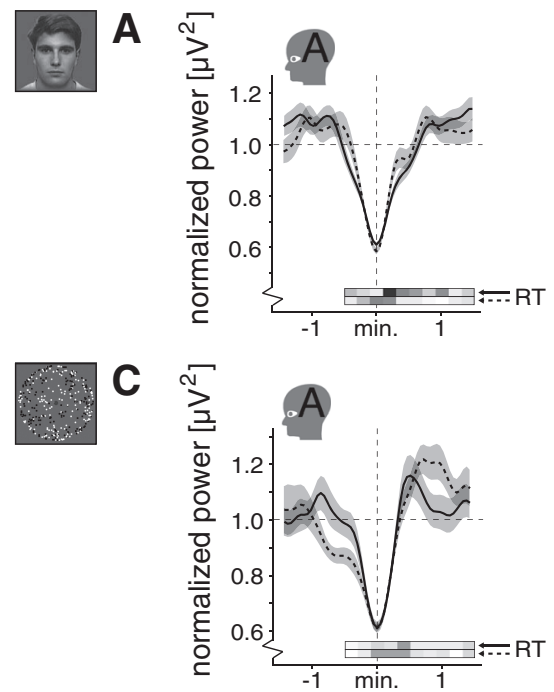
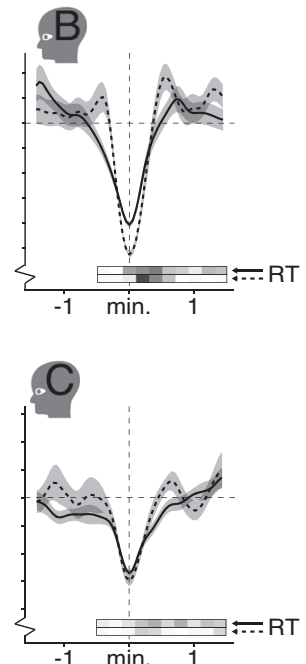

= spontaneous

..... = stimulus-induced

RT histogram:

0 25 reports

High-frequency perturbations (time-locked to peak)

B
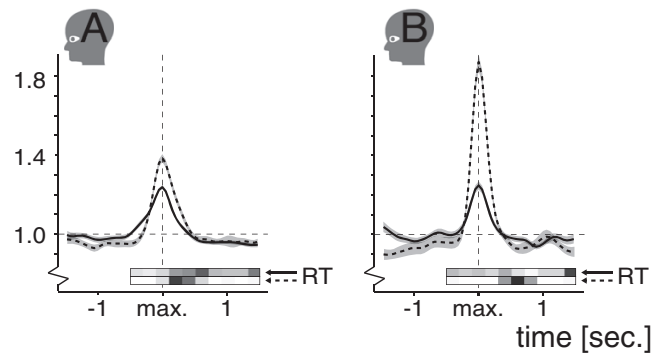

D
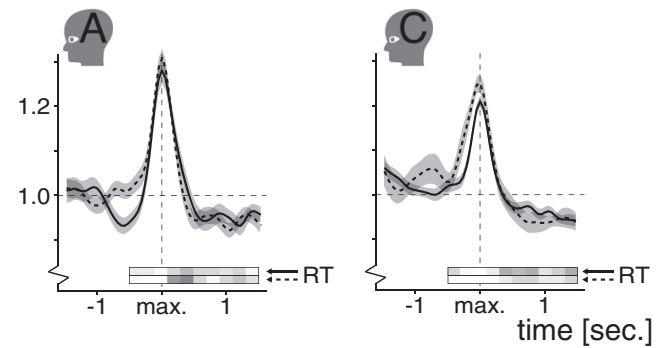

E
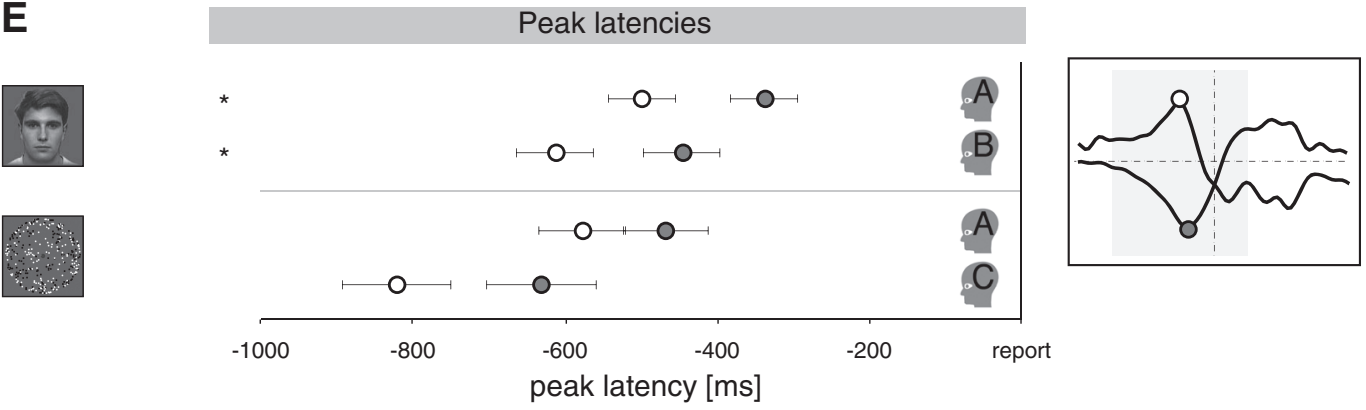

0 = low freq.

$0=$ high freq.

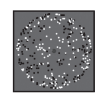

Figure 5. Peak-locked spectral perturbations and peak latencies for individual participants in both experiments. A, Spectral perturbations for the low-frequency band of interest in the binocular rivalry experiment, time-locked to latency of the peak amplitude per epoch [minimum (min.) within time window of interest]. These graphs are displayed for illustration purposes and are not used in any of the described analysis. By time-locking to the peak we can show perturbations without the influence of reaction-time jitter. The distribution of single-epoch reaction times relative to the peaks (RT) is indicated by the horizontal bars just below each graph, which represent histograms of the number of reports per time bin of $200 \mathrm{~ms}$ (top and bottom bar for spontaneous and stimulus-induced changes, respectively; darker gray means more reports). In comparison with the report-locked perturbations (Fig. 4), the peak-locked perturbations were similar for spontaneous and stimulus-induced changes, suggesting that (1) jitter in reaction times influenced the report-locked perturbations and (2) the spontaneous and stimulus-induced changes were associated with a similar occipital low-frequency power decrease. $\boldsymbol{B}$, Spectral perturbations for the high-frequency band of interest in the binocular rivalry experiment, time-locked to latency of the peak amplitude per epoch [maximum (max.) within time window of interest; further details as in $A$ ]. The high-frequency power increase was larger for stimulus-induced than for spontaneous changes. $C$, Same as $\boldsymbol{A}$, but here for the 3D-motion rivalry experiment. Again, spontaneous and stimulus-induced changes were associated with a similar low-frequency power decrease. $\boldsymbol{D}$, Same as $\boldsymbol{B}$, but here for 3D-motion rivalry experiment. Spontaneous and stimulus-induced changes were associated with a similar high-frequency power increase. $E$, For the individual participants, the average latency is shown ( \pm SEM across epochs) of the maximum high-frequency power (white dots) and the minimum low-frequency power (gray dots) within the time window of interest (see schematic illustration on the right), showing that the high-frequency power increase peaked earlier than the low-frequency power decrease. Values for spontaneous and stimulus-induced changes are averaged, as they did not differ. Each dot represents on average 90 epochs. ${ }^{*} p<0.05$ (see text).

the recordings of the individual occipital electrodes (electrode $X$ frequency band $x$ type of change ANOVA per participant; same

\section{$\leftarrow$}

(Figure legend continued.) Area of the low-frequency power decrease for the individual participants of the 3D-motion rivalry experiment, showing that the drop in low-frequency power was similar in magnitude for spontaneous and stimulus-induced changes. Details as in $\boldsymbol{A}$. $\boldsymbol{D}$, Top, Spectral perturbations for the high-frequency band of interest in the 3D-motion rivalry experiment, averaged across epochs (on average 34 epochs per line) for Participants A and C separately (presented on the left and right, respectively). Spontaneous and stimulus-induced changes were associated with a similar high-frequency power increase in the 3D-motion rivalry experiment, whereas in the binocular rivalry experiment $(\boldsymbol{B})$ the increase was smaller for spontaneous than for stimulus-induced changes. Bottom, Area of the high-frequency power increase for the individual participants of the 3D-motion rivalry experiment. Spontaneous and stimulus-induced changes were associated with a similar high-frequency power increase. Details as in $\boldsymbol{A}$. wavelet width for all frequencies) and found that the abovereported difference in latency between the frequency bands was systematic across electrodes (effect of frequency band: binocular rivalry experiment: both $F>6.5$; both $p \leq 0.01$; 3D-motion rivalry experiment: Participant $C, F_{(1,2108)}=12.8, p \approx 0$; Participant $\left.\mathrm{A}, F_{(1,2130)}=0.8, p=0.4\right)$. Also, it was larger for stimulusinduced than for spontaneous changes in Participants B and C (frequency band $\times$ type of change: both $F>5.3$; both $p \leq 0.02$ ), but not Participant A (both experiments: both $F \leq 1.9$; both $p \geq$ 0.2 ), an effect that was not observed in the averaged recordings. Spatial differences in peak latencies are described below.

\section{Interelectrode relations in power changes}

To get an overview of the spatial organization of the modulations described above, we calculated the areas of the power modula- 
A

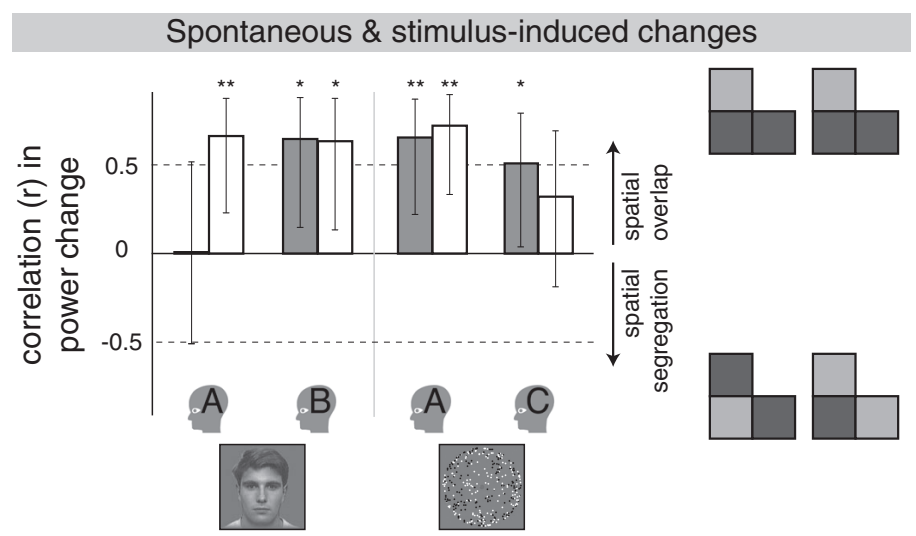

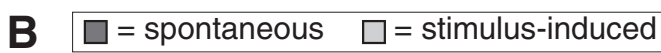

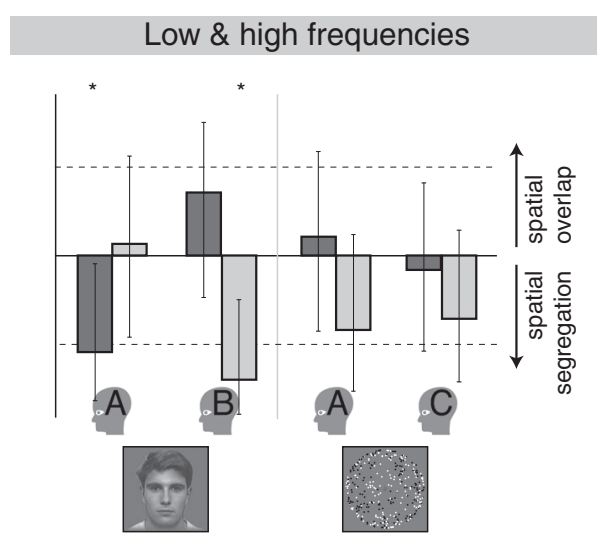

C

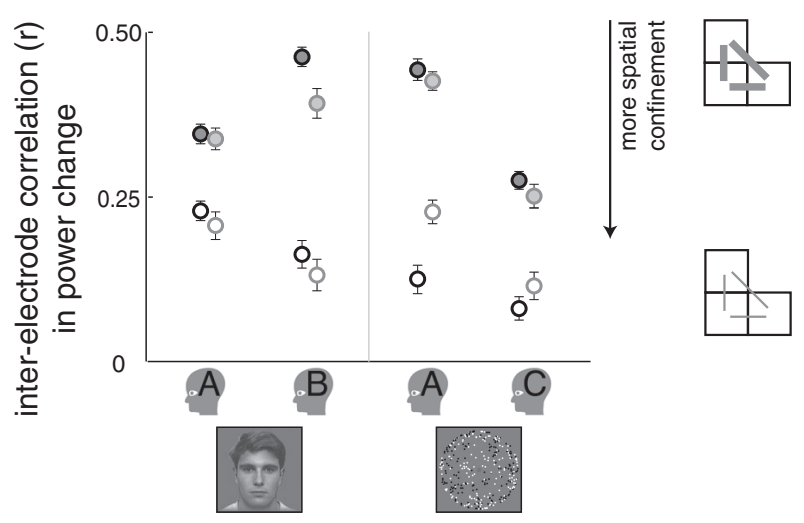

Figure 6. Measures of similarity in spatial organization. $\boldsymbol{A}$, The spatial correlation between spontaneous and stimulus-induced changes, presented for the individual participants. Positive correlation coefficients indicate that the pattern of activity across electrodes was similar (colocalized) for spontaneous and stimulus-induced changes (see schematic illustration on the right; icons symbolize activations of three neighboring electrodes). Specifically, the values ( $r \pm 95 \% \mathrm{Cl}$ ) represent the correlation in the areas of the modulations per electrode. Miniatures of the stimuli indicate the respective experiments. Small head icons indicate the participants. ${ }^{* *} p<0.01$; ${ }^{*} p<0.05$. Freq., Frequencies. $\boldsymbol{B}$, The spatial correlation between the low-frequency and high-frequency modulations, presented for the individual participants. A negative correlation coefficient indicates that electrodes with a large high-frequency modulation tended to have a small low-frequency modulation (correlation between high-frequency power increase as measured and low-frequency power decrease multiplied by -1 ). Details as in $\boldsymbol{A}$. $\boldsymbol{C}$, Interelectrode correlations for the low-frequency (filled circles) and high-frequency (open circles) modulation, presented for the individual participants. For spontaneous (circles with black edges) as well as the stimulus-induced (circles with gray edges) changes, the correlations were stronger in the low-frequency than in the high-frequency modulation, suggesting that for both types of changes the high-frequency modulation reflected more spatially confined activity than the low-frequency modulation. Specifically, the average correlation coefficients are presented (mean $r \pm$ SEM) of the correlations between all possible occipital electrode pairs regarding the areas of the modulations per epoch, as schematically illustrated by the icons on the right (gray lines symbolize correlations between pairs of electrodes).

tions in the recordings of the individual occipital electrodes (average across epochs) rather than their average. Across electrodes, power changes tended to correlate between spontaneous and stimulus-induced changes for all participants regarding the lowfrequency as well as the high-frequency modulation, suggesting colocalization of the activations associated with spontaneous and stimulus-induced changes (Fig. 6A; in the binocular rivalry experiment, some electrodes had a larger high-frequency modulation for stimulus-induced than for spontaneous changes; see next paragraph). For spontaneous as well as stimulus-induced changes, there was no systematic spatial relation between the low-frequency and high-frequency modulations, indicating that electrodes with a large drop in low-frequency power did not necessarily have a large increase in high-frequency power (Fig. 6B; correlations between high-frequency power increases as measured and low-frequency power decreases multiplied by -1 ).

In addition, we calculated the correlation of the power changes per electrode, per epoch, between all possible occipital electrode pairs (per participant, per type of change, per frequency band). For spontaneous as well as stimulus-induced changes, the interelectrode correlations were generally stronger in the lowfrequency than in the high-frequency modulation, supporting the idea that high-frequency activity is more spatially confined than low-frequency activity (Fig. 6C; type-of-change $\times$ frequency-band ANOVA performed per participant; frequency band: all $F \geq 40.4$, all $p \approx 0$ ).

\section{Spatial differences in power changes}

For all participants, we compared the activations of individual electrodes (electrode $\times$ type-of-change ANOVA per participant, per frequency band; we were interested in the electrode $X$ typeof-change interaction) and for Participant A, who completed both experiments, we compared individual electrode activations between the experiments (experiment $\times$ electrode ANOVA per frequency band, per type of change; we were interested in the electrode $\times$ experiment interaction). The above-described similarity between spontaneous and stimulus-induced changes regarding the low-frequency modulation (Fig. $4 A, B$ ) did not differ between electrodes (electrode $\times$ type-of-change interaction in both experiments and all participants: all $F \leq 0.7$, all $p>0.7)$ and 
A
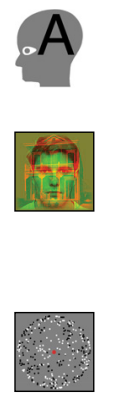
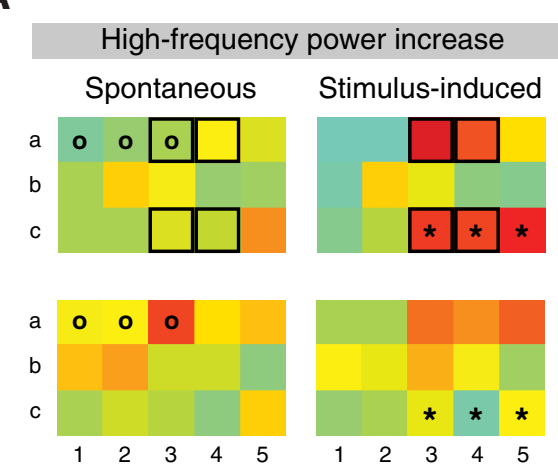

B
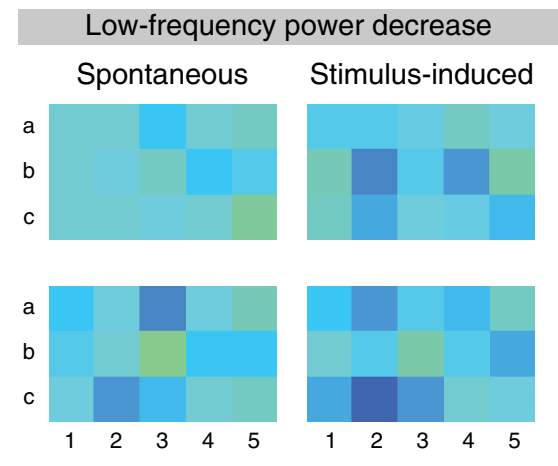

C
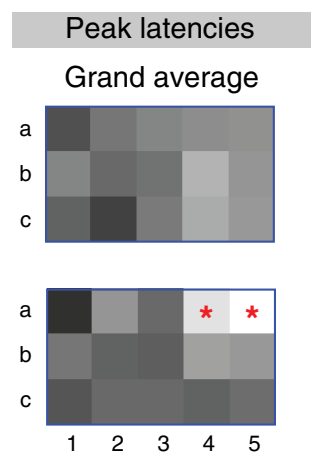

-175 mean $+175[\mathrm{~ms}]$

* = early peak

Figure 7. Recordings of individual occipital electrodes in Participant A. A, High-frequency power increase for individual electrodes (Fig. 1B, a1-c5) in Participant A (see color scale at bottom left). On a subset of the electrodes the high-frequency power increase in the binocular rivalry experiment was larger for stimulus-induced than for spontaneous changes (top row, black squares), a finding also reflected in the average across electrodes (Fig. 4B). Differences in individual-electrode activations between the experiments are also indicated (black asterisk: binocular $>3 D$-motion rivalry experiment; black circle: 3D-motion > binocular rivalry experiment). Miniatures of the stimuli indicate the respective experiments. Small head icon indicates the participant. $\boldsymbol{B}$, Low-frequency power decrease for individual electrodes in Participant A ( $A$, color scale). In neither of the experiments the low-frequency power decrease differed between electrodes, in line with the idea that the low-frequency modulation was less spatially confined than the high-frequency modulation (Fig. 6C). There were also no differences between the experiments in the individual-electrode activations. C, Peak latencies for individual electrodes in Participant A, averaged for the high-frequency and low-frequency modulation and both types of changes (mean latency per frequency band, per type of change, is subtracted; darker shades indicate a later peak; see scale at the bottom). In the 3D-motion (top graph) but not the binocular (bottom graph) rivalry experiment, two dorsal electrodes peaked early compared with other electrodes (indicated by red asterisks).

was thus not due to averaging out of opposing effects on individual electrodes. When comparing the experiments directly, Participant A also showed no differences between the experiments in the spatial organization of this modulation (Fig. $7 B$; experiment $\times$ electrode: $\left.F_{(14,2835)}=0.8, p=0.6\right)$.

There were differences between the experiments regarding the high-frequency modulation. For both participants in the 3Dmotion rivalry experiment, the above-described similarity between spontaneous and stimulus-induced changes (Fig. 4D) did not differ between electrodes (Fig. 7A, Participant A, bottom row; type of change $\times$ electrode: both $F<0.8$, both $p \leq 0.7)$. In the binocular rivalry experiment, by contrast, the high-frequency power increase was larger for stimulus-induced than for spontaneous changes (see above; Fig. $4 B$ ) and in both participants that completed this task this difference was confined to just a couple of electrodes (type of change $\times$ electrode: both $F>4.0$, both $p \approx 0$; then tested in partial ANOVAs per electrode). In both participants the electrodes carrying the difference were distributed unevenly across the electrode arrays, probably covering various functional regions rather than a single region (see below). A majority of the electrodes showed a similar high-frequency modulation for spontaneous and stimulus-induced changes (hence the colocalization reported above; Fig. 6A).

In Participant A there was a larger high-frequency modulation for stimulus-induced compared with spontaneous changes on dorsal electrodes a 3 and a 4 and ventral electrodes c3 and c4 [estimated functional regions: dorsal visual area (V) 2, dorsal V3, ventral V3, and ventral V4, respectively; Fig. 7A, top row]. Accordingly, these ventral electrodes ( $c 3$ and $c 4$ ) activated more to stimulus-induced changes in the binocular than in the $3 \mathrm{D}$ motion rivalry experiment (Fig. $7 A$; experiment $\times$ electrode: $F_{(14,2835)}=4.3, p \approx 0$; then tested in partial ANOVAs per electrode), as did ventral electrode c5 (estimated functional region: V8). Regarding spontaneous changes, dorsal electrodes a1, a2, and a3 (estimated functional region: V2 for all) activated more to
3D-motion than to binocular rivalry (Fig. $7 A$ ). Note that the estimated functional regions were not verified by functional mapping experiments and should therefore be taken with caution (see Materials and Methods). In Participant B there was a larger high-frequency modulation for stimulus-induced compared with spontaneous changes on electrodes b1, a2, b3, b4, and b7 (estimated functional regions: V2, V2, V3, V3, and V5/hMT + , respectively; electrode names in Fig. $1 B$; data not shown).

\section{Spatial differences in peak latencies}

Regarding Participant A, dorsal electrodes a4 and a5 (estimated functional regions: V3 and V5/hMT + , respectively; peak latencies: -694 and $-731 \mathrm{~ms}$, respectively) peaked early compared with other electrodes (peak latencies between -602 and -449 $\mathrm{ms}$ ) in the 3D-motion rivalry experiment (Fig. 7C; electrode: $\left.F_{(14,2130)}=2.4, p=0.003\right)$, but not in the binocular rivalry experiment (all peak latencies between -570 and $-412 \mathrm{~ms}$; effect of electrode: $F_{(14,3540)}=1.2, p=0.2$; electrode $\times$ frequency-band $\times$ type-of-change ANOVA per participant; we were interested in the main effect and interactions of electrode; same wavelet width used for all frequencies). The early activation was a general effect regardless of frequency band (electrode $\times$ frequency band: $F_{(14,2130)}=0.7, p=0.8$ ) or type of change (electrode $\times$ type of change: $\left.F_{(14,2130)}=0.9, p=0.6\right)$.

Regarding Participants B and C, there was a gradient present in the latencies per electrode, showing early activation of electrodes located centrally in the analyzed array (a4 and b4 for Participant B; c2 for Participant C; estimated functional regions: V3, V3, and V3A, respectively; peak latencies: $-581,-601$, and -745 $\mathrm{ms}$, respectively) and later activation of other electrodes (b1, b2, and b7 for Participant B; al and h3 for Participant C; peak latencies: $-485,-467,-397,-441$, and $-492 \mathrm{~ms}$, respectively; electrode names in Fig. $1 B$; electrode: both $F \geq 1.8$, both $p<0.05$; data not shown). For Participant B but none of the other participants, there was a significant electrode $\times$ frequency-band inter- 
action $\left(F_{(12,2600)}=3.0, p \approx 0\right)$, revealing that the difference in latency between the frequency bands reported above (Fig. $5 E$ ) was largest on electrodes b1, b3, and b4 (estimated functional regions: $\mathrm{V} 2, \mathrm{~V} 3$, and $\mathrm{V} 3$, respectively).

\section{Discussion}

The aim of the present study was to compare the extent to which regions in the occipital cortex sensitive to visual stimulation are transiently modulated in association with spontaneous and stimulus-induced perceptual changes (i.e., illusory and real changes in the stimulus, respectively). In line with previous literature, stimulus-induced perceptual changes were associated with a decrease in the power of low frequencies (theta, alpha, beta range) and an increase in the power of high frequencies (gamma range) in occipital cortex activity (Tallon-Baudry and Bertrand, 1999; Lachaux et al., 2005; Hoogenboom et al., 2006; Wilke et al., 2006; Siegel et al., 2007; Maier et al., 2008; Tallon-Baudry, 2009; Donner and Siegel, 2011). Interestingly, spontaneous perceptual changes were associated with a similar transient modulation of occipital activity (Fig. 3), despite the fact that these perceptual changes originated in the minds of the participants and lacked a concomitant change in the visual input.

\section{Transient decrease in low-frequency power}

In the binocular rivalry experiment (Fig. $4 A$ ), as well as in the 3D-motion rivalry experiment (Fig. $4 C$ ), we observed a transient drop in low-frequency power $(3-30 \mathrm{~Hz})$ preceding the report of both spontaneous and stimulus-induced perceptual changes. Following existing literature (Leopold and Logothetis, 1999; Sterzer et al., 2009), we initially aligned the epochs to the moment that the participant reported the perceived change (Fig. 4). Additionally, to assess the influence of reaction-time jitter, we performed two analyses largely insensitive to temporal jitter; namely, we calculated the area delimited by the report-locked changes in high-frequency and low-frequency power (Fig. 4, bar charts) and, by aligning epochs to peak latency before averaging across epochs, we obtained a visual representation of the perturbations unaffected by reaction-time jitter (for illustration purposes only; Fig. 5; see Materials and Methods). Whereas the low-frequency power decrease appeared to be smaller and wider for spontaneous compared with stimulus-induced changes in the averaged perturbations time-locked to the report (similar to scalp recordings reported by Strüber and Herrmann, 2002), the areas of the modulations and the peak-locked perturbations were similar for spontaneous and stimulus-induced changes (for binocular rivalry: Figs. $4 A, 5 A$; for 3D-motion rivalry: Figs. $4 C, 5 C$ ).

We suggest that the difference observed in the report-locked perturbations resulted from increased temporal uncertainty in the reports of the spontaneous compared with the stimulusinduced changes (Kornmeier and Bach, 2006; Knapen et al., 2011; Naber et al., 2011), because the transition periods between percepts can be less discrete in time for spontaneous than for stimulus-induced changes (Hollins and Hudnell, 1980; Anstis et al., 1985). However, we cannot exclude the possibility that the neural modulations themselves were more variable in time for the spontaneous changes. The similarity between spontaneous and stimulus-induced changes in the low-frequency modulation was widespread across the individual occipital electrodes and unrelated to cancelling-out of opposing effects between electrodes. We conclude that the transient drop in occipital low-frequency power was similar in magnitude for spontaneous and stimulusinduced changes.

\section{Transient increase in high-frequency power}

Spontaneous and stimulus-induced changes in perception were both associated with an increase in high-frequency power (50$130 \mathrm{~Hz}$ ). Previously reported scalp recordings revealed no highfrequency modulations for spontaneous changes (Müller et al., 1999; Strüber and Herrmann, 2002). The dispersion of neural signals measured on the scalp, by dura, skull, and skin, may have precluded detection of local high-frequency activity in these studies (Privman et al., 2007; Jerbi et al., 2009; Crone et al., 2011; Lachaux et al., 2012).

In the binocular rivalry experiment, the high-frequency modulation was smaller for spontaneous than for stimulus-induced changes. This difference was unrelated to a difference in temporal uncertainty because it was also observed in the areas of the modulation and the peak-locked perturbations (Figs. 4B, 5B). Analysis of individual occipital electrodes indicated that the difference was confined to a distributed subset of electrodes that covered various functional regions rather than a single region (Fig. 7A; Results). The stimulus-induced changes probably elicited a ubiquitous bottom-up response, whereas spontaneous changes may have recruited subsets of visual neurons specialized for the features of the stimulus (Haynes and Rees, 2005; de Jong et al., 2012), resulting in a smaller measured response when an electrode covers activated as well as silent neurons.

In the 3D-motion rivalry experiment, the high-frequency modulations associated with spontaneous and stimulus-induced changes were of a similar magnitude (Figs. 4D, 5D). In this experiment, stimulus-induced changes were quite subtle, involving only a change of motion direction in-and not the removal of-the perceived image (Fig. 2C). Thereby, spontaneous and stimulus-induced changes may have engaged the same dorsal regions sensitive to global motion and motion in depth (Paradis et al., 2000; Brouwer and van Ee, 2007); hence, the similar highfrequency modulations.

\section{Spatial overlap between spontaneous and stimulus- induced changes}

There has been an extensive debate in the literature regarding the role of the occipital cortex in spontaneous perceptual changes: either the occipital cortex is recruited for the maintenance of a percept by means of feedback from cognitive regions, or perceptual changes are initiated within the occipital cortex and communicated to higher-level regions in a bottom-up fashion (see Introduction). We reasoned that, while both views are compatible with sustained activity differences between the two possible perceptual states (as found previously; see Introduction), only the latter view implicates that transient activations at the moment of a perceptual change are present in the occipital cortex.

Accordingly, we observed transient neural modulations in the occipital cortex that differed little or not at all between stimulusinduced and spontaneous perceptual changes (Figs. 4, 5) and that spatially overlapped (Fig. 6A). We thus suggest that the spontaneous perceptual changes are initiated within-rather than beyond-the occipital cortex. However, this may not be a strictly bottom-up process, since our results are compatible with the idea that feedback activity within visual networks is necessary for the initiation of spontaneous perceptual changes (Tong et al., 2006; Wolf and Hochstein, 2011; as discussed in the next section). Investigations at a finer spatial scale are needed to gain knowledge about the neural interactions underlying the observed modulations and may also reveal critical differences between spontaneous and stimulus-induced changes. It could be that spontaneous changes are associated with more competitive neural interactions 
than stimulus-induced changes, possibly through the activation of inhibitory interneurons within stimulus-specific visual regions (Blake and Logothetis, 2002; Long and Toppino, 2004).

Transient activations of parietal and frontal regions reported previously in association with spontaneous changes (see Introduction) may be related to cognitive processes associated with perception, such as attention, intention, introspection, or perceptual uncertainty, instead of reflecting initiation of perceptual changes (Raemaekers et al., 2009; Knapen et al., 2011; de Graaf et al., 2011; Frässle et al., 2014; Brascamp et al., 2015). The occipital perturbations we presently observed are similar to previously reported perturbations elicited by changes in visual stimulation (see above) and not similar to perturbations previously reported in association with visual attention (Kelly et al., 2006; Doesburg et al., 2016). Nevertheless, it is possible that secondary effects, for example a change in attention following a perceptual change, also contributed to the present results (Wolf and Hochstein, 2011; Blake et al., 2014).

\section{Functional dissociation between low and high frequencies}

It has often been reported that high-frequency modulations reflect fast, local processes, whereas low-frequency modulations reflect slower, global processes (Kopell et al., 2000; von Stein and Sarnthein, 2000; Buzsáki and Draguhn, 2004; Tallon-Baudry, 2009; Donner and Siegel, 2011). Accordingly, we found that the high-frequency modulation peaked earlier (Fig. 5E; Wilke et al., 2006) and correlated less between occipital electrodes than the low-frequency modulation (Fig. 6). The high-frequency and low-frequency modulation further differed regarding which electrodes showed a large or small modulation (Fig. 6A,B), evidencing a difference in spatial organization. Taking the above into consideration, we tentatively propose the following functional dissociation between the frequency bands: whereas the high-frequency modulation reflects the initiation of perceptual changes in specialized visual networks, the low-frequency modulation reflects distribution of change-related activity to a wider range of visual networks, thereby mediating the maintenance of the perceptual state.

This proposal does not explain our finding that, regardless of the frequency band, some electrodes activated earlier than others (Fig. 7C). This finding suggests a coupling of the lowfrequency and high-frequency modulation in time, superimposed on the latency difference that was present across electrodes and contrasting the dissociation in terms of spatial organization. However, in line with the idea, neural modulations reflecting (the maintenance of) the perceptual state have primarily been reported in low-frequency rather than highfrequency occipital activity (Gail et al., 2004; Maier et al., 2008; but see Matsuzaki et al., 2012; Sandberg et al., 2014; Vidal et al., 2014). The transient nature of the current lowfrequency modulation suggests it may not reflect ongoing maintenance of a percept, but rather could instigate coordination of distributed activity within visual cortex needed for maintenance. The proposed role of the high-frequency modulation is congruent with findings that features of the visual input are more reliably reflected in high-frequency than in low-frequency activity (Frien and Eckhorn, 2000; Hall et al., 2005; Henrie and Shapley, 2005; Liu and Newsome, 2006; Siegel et al., 2007; Belitski et al., 2008; Donner and Siegel, 2011). Furthermore, the idea of local initiation and more widespread maintenance of percepts is compatible with a previous fMRI study that decomposed spatial patterns of occipital activity and reported that the prevalence of spontaneous changes was reflected locally in $\mathrm{V} 4$, whereas the duration of percepts was reflected in multiple occipital regions (areas V1V3; Donner et al., 2013).

Regardless of the proposed functional dissociation between low-frequency and high-frequency modulations, our results do not point to a bottom-up processing strategy for spontaneous perceptual changes. First, we found that some electrodes activated earlier than others, but there was no bottom-up spatial gradient present in these latencies (Fig. 7C; Results). Second, the high-frequency modulation was confined to a subset of electrodes, but these covered various functional regions rather than a single low-level region (Fig. 7A; Results). Feedback and distributed activity within visual networks thus seems necessary for the initiation of spontaneous perceptual changes (Tong et al., 2006).

\section{Considerations and limitations}

Although the analyzed occipital electrodes did not overlap with the focus of epileptic seizures (see Materials and Methods), it remains uncertain whether-and how-the epileptic condition of the participants affected the results. Also, different results might have been obtained from parts of the occipital cortex that were not sampled. Furthermore, it has been reported that eye movements may influence oscillatory neural activity in monkeys (Bosman et al., 2009). We cannot rule out the possibility that eye movements contributed to our results, including those related to both stimulus-induced and spontaneous perceptual changes, although previous experiments with a similar setup and fixation instructions showed that spontaneous perceptual changes occur independently of eye movements (van Dam and van Ee, 2005; Brouwer and van Ee, 2007). Finally, due to the possibility of passive volume conduction, electrical activity may originate in regions distant from the recording electrode. However, the interelectrode differences we report regarding the high-frequency modulation (Fig. $6 C$, correlations; Fig. $7 A$, power changes) are in line with the idea of local or nearby sources for this modulation. Furthermore, a previous intracranial study indicated that broadband oscillatory activity in human visual cortex reflected local sources (Winawer et al., 2013).

\section{Conclusion}

We observed transient neural modulations in the occipital cortex that differed little or not at all between stimulus-induced and spontaneous perceptual changes, even though the latter lack a concomitant change in the visual stimulus. We tentatively suggest that the observed increase in occipital high-frequency power reflects the initiation of perceptual changes, whereas the drop in occipital low-frequency power, which was later in time and less spatially confined, reflects their distribution. We argue that the spontaneous initiation of new percepts can occur within (rather than beyond) the visual cortex. Furthermore, this likely involves distributed activity and feedback within visual cortex, as our results do not support a mere bottom-up processing strategy.

\section{References}

Anstis S, Giaschi D, Cogan AI (1985) Adaptation to apparent motion. Vision Res 25:1051-1062. CrossRef Medline

Başar-Eroglu C, Strüber D, Kruse P, Başar E, Stadler M (1996) Frontal gamma-band enhancement during multistable visual perception. Int J Psychophysiol 24:113-125. CrossRef Medline

Belitski A, Gretton A, Magri C, Murayama Y, Montemurro MA, Logothetis NK, Panzeri S (2008) Low-frequency local field potentials and spikes in primary visual cortex convey independent visual information. J Neurosci 28:5696-5709. CrossRef Medline 
Blake R, Logothetis N (2002) Visual competition. Nat Rev Neurosci 3: 13-21. CrossRef Medline

Blake R, Brascamp J, Heeger DJ (2014) Can binocular rivalry reveal neural correlates of consciousness? Philos Trans R Soc Lond B Biol Sci 369: 20130211. CrossRef

Bosman CA, Womelsdorf T, Desimone R, Fries P (2009) A microsaccadic rhythm modulates gamma-band synchronization and behavior. J Neurosci 290:9471-9480.

Brascamp J, Blake R, Knapen T (2015) Negligible fronto-parietal BOLD activity accompanying unreportable switches in bistable perception. Nat Neurosci 18:1672-1678. CrossRef Medline

Braunstein M (1977) Perceived direction of rotation of simulated threedimensional patterns. Percept Psychophys 21:553-557. CrossRef

Brouwer GJ, van Ee R (2006) Endogenous influences on perceptual bistability depend on exogenous stimulus characteristics. Vision Res 46:3393-3402. CrossRef Medline

Brouwer GJ, van Ee R (2007) Visual cortex allows prediction of perceptual states during ambiguous structure-from-motion. J Neurosci 27: 1015-1023. CrossRef Medline

Buzsáki G, Draguhn A (2004) Neuronal oscillations in cortical networks. Science 304:1926-1929. CrossRef Medline

Crone NE, Korzeniewska A, Franaszczuk PJ (2011) Cortical gamma responses: searching high and low. Int J Psychophysiol 79:9-15. CrossRef Medline

de Graaf TA, de Jong MC, Goebel R, van Ee R, Sack AT (2011) On the functional relevance of frontal cortex for passive and voluntarily controlled bistable vision. Cereb Cortex 21:2322-2331. CrossRef Medline

de Jong MC, Kourtzi Z, van Ee R (2012) Perceptual experience modulates cortical circuits involved in visual awareness. Eur J Neurosci 36: 3718-3731. CrossRef Medline

de Jong MC, Brascamp JW, Kemner C, van Ee R, Verstraten FA (2014) Implicit perceptual memory modulates early visual processing of ambiguous images. J Neurosci 34:9970-9981. CrossRef Medline

Delorme A, Makeig S (2004) EEGLAB: an open source toolbox for analysis of single-trial EEG dynamics including independent component analysis. J Neurosci Methods 134:9-21. CrossRef Medline

Doesburg SM, Kitajo K, Ward LM (2005) Increased gamma-band synchrony precedes switching of conscious perceptual objects in binocular rivalry. Neuroreport 16:1139-1142. CrossRef Medline

Doesburg SM, Bedo N, Ward LM (2016) Top-down alpha oscillatory network interactions during visuospatial attention orienting. Neuroimage 132:512-519. CrossRef Medline

Donner TH, Siegel M (2011) A framework for local cortical oscillation patterns. Trends Cogn Sci 15:191-199. CrossRef Medline

Donner TH, Sagi D, Bonneh YS, Heeger DJ (2013) Retinotopic patterns of correlated fluctuations in visual cortex reflect the dynamics of spontaneous perceptual suppression. J Neurosci 33:2188-2198. CrossRef Medline

Ehm W, Bach M, Kornmeier J (2011) Ambiguous figures and binding: EEG frequency modulations during multistable perception. Psychophysiology 48:547-558. CrossRef Medline

Frässle S, Sommer J, Jansen A, Naber M, Einhäuser W (2014) Binocular rivalry: frontal activity relates to introspection and action but not to perception. J Neurosci 34:1738-1747. CrossRef Medline

Frien A, Eckhorn R (2000) Functional coupling shows stronger stimulus dependency for fast oscillations than for low-frequency components in striate cortex of awake monkey. Eur J Neurosci 12:1466-1478. CrossRef Medline

Gail A, Brinksmeyer HJ, Eckhorn R (2004) Perception-related modulations of local field potential power and coherence in primary visual cortex of awake monkey during binocular rivalry. Cereb Cortex 14:300-313. CrossRef Medline

Hall SD, Holliday IE, Hillebrand A, Singh KD, Furlong PL, Hadjipapas A, Barnes GR (2005) The missing link: analogous human and primate cortical gamma oscillations. Neuroimage 26:13-17. CrossRef Medline

Haynes JD, Rees G (2005) Predicting the stream of consciousness from activity in human visual cortex. Curr Biol 15:1301-1307. CrossRef Medline

Henrie JA, Shapley R (2005) LFP power spectra in V1 cortex: the graded effect of stimulus contrast. J Neurophysiol 94:479-490. CrossRef Medline

Hermes D, Miller KJ, Noordmans HJ, Vansteensel MJ, Ramsey NF (2010) Automated electro-corticographic electrode localization on individual rendered brain surfaces. J Neurosci Methods 185:293-298. CrossRef Medline

Hol K, Koene A, van Ee R (2003) Attention-biased multi-stable surface perception in three-dimensional structure-from-motion. J Vis 3(7): 486-498. Medline

Hollins M, Hudnell K (1980) Adaptation of the binocular rivalry mechanism. Invest Ophthalmol Vis Sci 19:1117-1120. Medline

Hoogenboom N, Schoffelen JM, Oostenveld R, Parkes LM, Fries P (2006) Localizing human visual gamma-band activity in frequency, time and space. Neuroimage 29:764-773. CrossRef Medline

Jerbi K, Ossandón T, HamaméCM, Senova S, Dalal SS, Jung J, Minotti L, Bertrand O, Berthoz A, Kahane P, Lachaux JP (2009) Task-related gamma-band dynamics from an intracerebral perspective: review and implications for surface EEG and MEG. Hum Brain Mapp 30:1758-1771. CrossRef Medline

Kelly SP, Lalor EC, Reilly RB, Foxe JJ (2006) Increases in alpha oscillatory power reflect an active retinotopic mechanism for distracter suppression during sustained visuospatial attention. J Neurophysiol 95:3844-3851. CrossRef Medline

Kleinschmidt A, Büchel C, Zeki S, Frackowiak RS (1998) Human brain activity during spontaneously reversing perception of ambiguous figures. Proc Biol Sci 265:2427-2433. CrossRef Medline

Knapen T, Brascamp J, Pearson J, van Ee R, Blake R (2011) The role of frontal and parietal brain areas in bistable perception. J Neurosci 31: 10293-102301. CrossRef Medline

Kopell N, Ermentrout GB, Whittington MA, Traub RD (2000) Gamma rhythms and beta rhythms have different synchronization properties. Proc Natl Acad Sci U S A 97:1867-1872. CrossRef Medline

Kornmeier J, Bach M (2006) Bistable perception-along the processing chain from ambiguous visual input to a stable percept. Int J Psychophysiol 62:345-349. CrossRef Medline

Lachaux JP, George N, Tallon-Baudry C, Martinerie J, Hugueville L, Minotti L, Kahane P, Renault B (2005) The many faces of the gamma band response to complex visual stimuli. Neuroimage 25:491-501. CrossRef Medline

Lachaux JP, Axmacher N, Mormann F, Halgren E, Crone NE (2012) High-frequency neural activity and human cognition: past, present and possible future of intracranial EEG research. Prog Neurobiol 98: 279-301. CrossRef Medline

Lee SH, Blake R, Heeger DJ (2007) Hierarchy of cortical responses underlying binocular rivalry. Nat Neurosci 10:1048-1054. CrossRef Medline

Leopold DA, Logothetis NK (1996) Activity changes in early visual cortex reflect monkeys' percepts during binocular rivalry. Nature 379:549-553. CrossRef Medline

Leopold DA, Logothetis NK (1999) Multistable phenomena: changing views in perception. Trends Cogn Sci 3:254-264. CrossRef Medline

Levelt WJM (1966) The alternation process in binocular rivalry. Brit J Psychol 57:225-238. CrossRef

Liu J, Newsome WT (2006) Local field potential in cortical area MT: stimulus tuning and behavioral correlations. J Neurosci 26:7779-7790. CrossRef Medline

Long GM, Toppino TC (2004) Enduring interest in perceptual ambiguity: alternating views of reversible figures. Psychol Bull 130:748-768. CrossRef Medline

Luck SJ (2005) An introduction to the event-related potential technique. Cambridge, MA: MIT.

Lumer ED, Friston KJ, Rees G (1998) Neural correlates of perceptual rivalry in the human brain. Science 280:1930-1934. CrossRef Medline

Maier A, Wilke M, Aura C, Zhu C, Ye FQ, Leopold DA (2008) Divergence of fMRI and neural signals in V1 during perceptual suppression in the awake monkey. Nat Neurosci 11:1193-1200. CrossRef Medline

Matsuzaki N, Juhász C, Asano E (2012) Oscillatory modulations in human fusiform cortex during motion-induced blindness: intracranial recording. Clin Neurophysiol 123:1925-1930. CrossRef Medline

Müller TJ, Federspiel A, Fallgatter AJ, Strik WK (1999) EEG signs of vigilance fluctuations preceding perceptual flips in multistable illusionary motion. Neuroreport 10:3423-3427. CrossRef Medline

Naber M, Frässle S, Einhäuser (2011) Perceptual rivalry: reflexes reveal the gradual nature of visual awareness. PLoS One 6:e20910. CrossRef Medline

Paradis AL, Cornilleau-Pérès V, Droulez J, Van De Moortele PF, Lobel E, Berthoz A, Le Bihan D, Poline JB (2000) Visual perception of motion 
and 3-D structure from motion: an fMRI study. Cereb Cortex 10: 772-783. CrossRef Medline

Polonsky A, Blake R, Braun J, Heeger DJ (2000) Neuronal activity in human primary visual cortex correlates with perception during binocular rivalry. Nat Neurosci 3:1153-1159. CrossRef Medline

Privman E, Nir Y, Kramer U, Kipervasser S, Andelman F, Neufeld MY, Mukamel R, Yeshurun Y, Fried I, Malach R (2007) Enhanced category tuning revealed by intracranial electroencephalograms in high-order human visual areas. J Neurosci 27:6234-6242. CrossRef Medline

Raemaekers M, van der Schaaf ME, van Ee R, van Wezel RJ (2009) Widespread fMRI activity differences between perceptual states in visual rivalry are correlated with differences in observer biases. Brain Res 1252: 161-171. CrossRef Medline

Sandberg K, Bahrami B, Kanai R, Barnes GR, Overgaard M, Rees G (2013) Early visual responses predict conscious face perception within and between subjects during binocular rivalry. J Cogn Neurosci 25:969-985. CrossRef Medline

Sandberg K, Barnes GR, Bahrami B, Kanai R, Overgaard M, Rees G (2014) Distinct MEG correlates of conscious experience, perceptual reversals and stabilization during binocular rivalry. Neuroimage 100:161-175. CrossRef Medline

Siegel M, Donner TH, Oostenveld R, Fries P, Engel AK (2007) Highfrequency activity in human visual cortex is modulated by visual motion strength. Cereb Cortex 17:732-741. Medline

Sterzer P, Kleinschmidt A (2007) A neural basis for inference in perceptual ambiguity. Proc Natl Acad Sci U S A 104:323-328. CrossRef Medline

Sterzer P, Kleinschmidt A, Rees G (2009) The neural bases of multistable perception. Trends Cogn Sci 13:310-318. CrossRef Medline

Strüber D, Herrmann CS (2002) MEG alpha activity decrease reflects destabilization of multistable percepts. Brain Res Cogn Brain Res 14:370-382. CrossRef Medline

Tallon-Baudry C (2009) The roles of gamma-band oscillatory synchrony in human visual cognition. Front Biosci (Landmark Ed) 14:321-332. Medline

Tallon-Baudry C, Bertrand O (1999) Oscillatory gamma activity in humans and its role in object representation. Trends Cogn Sci 3:151-162. CrossRef Medline

Tong F, Engel SA (2001) Interocular rivalry revealed in the human cortical blind-spot representation. Nature 411:195-199. CrossRef Medline

Tong F, Meng M, Blake R (2006) Neural bases of binocular rivalry. Trends Cogn Sci 10:502-511. CrossRef Medline

van Dam LC, van Ee R (2005) The role of (micro)saccades and blinks in perceptual bi-stability from slant rivalry. Vision Res 45:2417-2435. CrossRef Medline

Vidal JR, Perrone-Bertolotti M, Kahane P, Lachaux JP (2014) Intracranial spectral amplitude dynamics of perceptual suppression in fronto-insular, occipito-temporal, and primary visual cortex. Front Psychol 5:1545. CrossRef Medline

von Stein A, Sarnthein J (2000) Different frequencies for different scales of cortical integration: from local gamma to long range alpha/theta synchronization. Int J Psychophysiol 38:301-313. CrossRef Medline

Wallach H, O'Connell DN (1953) The kinetic depth effect. J Exp Psychol 45:205-217. CrossRef Medline

Wilke M, Logothetis NK, Leopold DA (2006) Local field potential reflects perceptual suppression in monkey visual cortex. Proc Natl Acad Sci U S A 103:17507-17512. CrossRef Medline

Winawer J, Kay KN, Foster BL, Rauschecker AM, Parvizi J, Wandell BA (2013) Asynchronous broadband signals are the principal source of the BOLD response in human visual cortex. Curr Biol 23:1145-1153. CrossRef Medline

Wolf M, Hochstein S (2011) High-level binocular rivalry effects. Front Hum Neurosci 5:129. CrossRef Medline

Xu H, Han C, Chen M, Li P, Zhu S, Fang Y, Hu J, Ma H, Lu HD (2016) Rivalry-like neural activity in primary visual cortex in anesthetized monkeys. J Neurosci 36:3231-3242. CrossRef Medline 